OPEN ACCESS

Edited by:

Rey Carabeo,

Washington State University, USA

Reviewed by:

Eric Ghigo,

Centre National de la Recherche

Scientifique, France

Elizabeth Ann Rucks,

University of South Dakota, USA

*Correspondence:

Jason A. Carlyon

jason.carlyon@vcuhealth.org

${ }^{\dagger}$ Present Address:

Hilary K. Truchan,

Department of Microbiology and Immunology, Northwestern University

Medical School, Chicago, IL, USA

Received: 23 September 2015 Accepted: 08 February 2016 Published: 01 March 2016

Citation:

Truchan HK, Cockburn CL, Hebert KS, Magunda F, Noh SM and

Carlyon JA (2016) The

Pathogen-Occupied Vacuoles of

Anaplasma phagocytophilum and Anaplasma marginale Interact with the

Endoplasmic Reticulum.

Front. Cell. Infect. Microbiol. 6:22.

doi: 10.3389/fcimb.2016.00022

\section{The Pathogen-Occupied Vacuoles of Anaplasma phagocytophilum and Anaplasma marginale Interact with the Endoplasmic Reticulum}

\author{
Hilary K. Truchan ${ }^{1+}$, Chelsea L. Cockburn ${ }^{1}$, Kathryn S. Hebert ${ }^{1}$, Forgivemore Magunda ${ }^{2,3}$, \\ Susan M. Noh ${ }^{2,4}$ and Jason A. Carlyon ${ }^{1 *}$ \\ ' Department of Microbiology and Immunology, Virginia Commonwealth University School of Medicine, Richmond, VA, USA, \\ ${ }^{2}$ Program in Vector Borne Diseases, Department of Veterinary Microbiology and Pathology, Washington State University, \\ Pullman, WA, USA, ${ }^{3}$ The Paul G. Allen School for Global Animal Health, Washington State University, Pullman, WA, USA, \\ ${ }^{4}$ Animal Disease Research Unit, Agricultural Research Service, U. S. Department of Agriculture, Pullman, WA, USA
}

The genus Anaplasma consists of tick-transmitted obligate intracellular bacteria that invade white or red blood cells to cause debilitating and potentially fatal infections. A. phagocytophilum, a human and veterinary pathogen, infects neutrophils to cause granulocytic anaplasmosis. A. marginale invades bovine erythrocytes. Evidence suggests that both species may also infect endothelial cells in vivo. In mammalian and arthropod host cells, A. phagocytophilum and A. marginale reside in host cell derived pathogen-occupied vacuoles (POVs). While it was recently demonstrated that the A. phagocytophilum-occupied vacuole (ApV) intercepts membrane traffic from the trans-Golgi network, it is unclear if it or the A. marginale-occupied vacuole (AmV) interacts with other secretory organelles. Here, we demonstrate that the ApV and AmV extensively interact with the host endoplasmic reticulum (ER) in endothelial, myeloid, and/or tick cells. ER lumen markers, calreticulin, and protein disulfide isomerase, and the ER membrane marker, derlin-1, were pronouncedly recruited to the peripheries of both POVs. ApV association with the ER initiated early and continued throughout the infection cycle. Both the ApV and AmV interacted with the rough ER and smooth ER. However, only derlin-1-positive rough ER derived vesicles were delivered into the ApV lumen where they localized with intravacuolar bacteria. Transmission electron microscopy identified multiple ER-POV membrane contact sites on the cytosolic faces of both species' vacuoles that corresponded to areas on the vacuoles' lumenal faces where intravacuolar Anaplasma organisms closely associated. A. phagocytophilum is known to hijack Rab10, a GTPase that regulates ER dynamics and morphology. Yet, ApVER interactions were unhindered in cells in which Rab10 had been knocked down, demonstrating that the GTPase is dispensable for the bacterium to parasitize the ER. These data establish the ApV and AmV as pathogen-host interfaces that directly engage the ER in vertebrate and invertebrate host cells and evidence the conservation of ER parasitism between two Anaplasma species.

Keywords: Anaplasmataceae, Rickettsia, intracellular bacteria, endoplasmic reticulum, Rab, pathogen synapse 


\section{INTRODUCTION}

Anaplasma phagocytophilum and Anaplasma marginale are ticktransmitted obligate intracellular bacterial pathogens of the Family Anaplasmataceae that cause debilitating and potentially fatal diseases (Carlyon, 2012). A. phagocytophilum infects neutrophils to cause granulocytic anaplasmosis in humans and animals (Truchan et al., 2013). Over the decade leading up to 2012, the most recent year for which United States Centers for Disease Control (CDC) statistics are available, the number of human granulocytic anaplasmosis (HGA) cases reported annually to the $\mathrm{CDC}$ rose nearly seven-fold (CDC, 2013). The disease also continues to emerge in Europe and Asia (Truchan et al., 2013). HGA is an acute febrile illness that can be accompanied by non-specific symptoms including headache, malaise, myalgia, elevated liver enzymes, leukopenia, and thrombocytopenia (Truchan et al., 2013). A. marginale is a strict bovine pathogen that is endemic throughout the southern Atlantic, Gulf coast, and several Midwestern and Western U. S. states, as well as Mexico, Central and South America, and the Caribbean Islands. It infects erythrocytes, which can result in anemia, weight loss, reduced growth, and milk production, and abortion in pregnant cattle (Kocan et al., 2010; Suarez and Noh, 2011). Following resolution of acute disease, bovine anaplasmosis can remain chronic for the life of the animal and is estimated to cost the U.S. and South American cattle industries hundreds of millions of dollars each year (Kocan et al., 2003; Suarez and Noh, 2011).

A. phagocytophilum and A. marginale replicate within host cell-derived vacuoles (Carlyon, 2012). Both will infect mammalian and tick cell lines, including human promyelocytic HL-60 cells (A. phagocytophilum only), Ixodes scapularis embryonic ISE6 cells (both Anaplasma spp.), and primate RF/6A endothelial cells (both species) (Goodman et al., 1996; Woldehiwet et al., 2002; Munderloh et al., 2004; Zivkovic et al., 2009). RF/6A cells are particularly useful models for studying the cellular microbiology of these bacteria because they are large and flat, making them ideal for imaging (Munderloh et al., 2004; Sukumaran et al., 2011; Beyer et al., 2014; Truchan et al., 2016). Moreover, A. phagocytophilum and A. marginale have been detected in endothelial cells of tissue sections recovered from experimentally infected animals (Herron et al., 2005; Wamsley et al., 2011). During growth in tissue culture cells, both Anaplasma spp. cycle between a dense-cored (DC) morphotype that binds and invades host cells and a reticulate cell (RC) morphotype that replicates inside the pathogen-occupied vacuole (POV) (Munderloh et al., 2004; Troese and Carlyon, 2009). Like many professional vacuolar bacterial pathogens (Brumell and Scidmore, 2007; Sherwood and Roy, 2013), A. phagocytophilum selectively recruits a subset of Rab GTPases to its vacuole (Huang et al., 2010a). We recently reported that one such GTPase, Rab10, is critical for the pathogen to parasitize exocytic traffic from the trans-Golgi network (Truchan et al., 2016). Whether the A. phagocytophilum-occupied vacuole (ApV) or A. marginale-occupied vacuole $(\mathrm{AmV})$ hijacks other arms of the secretory pathway in mammalian or tick host cells is unknown.
Nascent proteins that are destined for either secretion or for the plasma membrane, secretory, or endocytic organelles are first translocated into the endoplasmic reticulum (ER) where they are processed and subjected to quality control. Once thought of as a single large organelle, the ER is actually an assemblage of several membrane domains-the ribosome studded rough ER (RER), smooth ER (SER), mitochondria associated membrane (MAM), ER exit sites (ERESs), and ER quality control compartment (ERQC) (Lynes and Simmen, 2011; Benyair et al., 2015). Proteins are synthesized, Nterminally glycosylated, and translocated into the RER. Here, the glycoproteins are initially processed and begin the quality control process wherein they are cycled among the RER, SER, MAM, and ERQC. Properly folded secretory glycoproteins are segregated into ERESs, transported to the ERGIC (ER-to-Golgi intermediate compartment) and subsequently to the Golgi. In the ERQC, terminally misfolded glycoproteins are targeted for ER associated degradation (ERAD), a process by which the misfolded proteins are retrotranslocated to the cytosol, where they are degraded by the ubiquitin proteasome system (Benyair et al., 2015).

Rab10, which is important for A. phagocytophilum TGN parasitism (Truchan et al., 2016), not only directs exocytic traffic from the TGN (Liu and Storrie, 2012) but also regulates ER dynamics and morphology (English and Voeltz, 2013). Moreover, Rab1, a GTPase that directs vesicular traffic from the ER to the Golgi apparatus (Stenmark, 2009), is also recruited to the ApV (Huang et al., 2010a). Given these phenomena, the paucity of information on AmV-host cell interactions, and the dearth of knowledge on the cellular microbiology of Anaplasma spp. in tick cells, we investigated if the ApV and AmV engage the ER during infection of mammalian and tick host cells. Our data reveal that both POVs interact with the ER and that derlin-1-positive vesicles are delivered into their lumen. Thus, the ability to hijack the secretory pathway is conserved between A. phagocytophilum and A. marginale.

\section{MATERIALS AND METHODS}

\section{Cultivation of Uninfected and Anaplasma spp. Infected Cell Lines}

Uninfected and $A$. phagocytophilum (NCH-1 strain)-infected human promyelocytic HL-60 cells (CCL-240; American Type Culture Collections [ATCC, Manassas, VA]), RF/6A rhesus monkey choroidal endothelial cells (CRL-1780, ATCC), and ISE6 cells were cultured as described (Huang et al., 2010a, 2012; Beyer et al., 2014). A. marginale (St. Maries strain)infected RF/6A cells and uninfected and A. marginale infected ISE6 cells were gifts from Ulrike Munderloh (University of Minnesota, Minneapolis, MN). A. marginale infected ISE6 cells were cultured identically to A. phagocytophilum infected ISE6 cells. A. marginale infected $\mathrm{RF} / 6 \mathrm{~A}$ cells were maintained in $25 \mathrm{~cm}^{2}$ cell culture flasks as follows. When $\geq 80 \%$ of the cells had lysed and the media contained infectious $A$. marginale organisms, $5 \mathrm{ml}$ of the bacteria laden media was transferred to a $25 \mathrm{~cm}^{2}$ cell culture flask containing naïve $\mathrm{RF} / 6 \mathrm{~A}$ cells that were nearly confluent. Uninfected RF/6A cells were grown to near confluency 
in $25 \mathrm{~cm}^{2}$ cell culture flasks. Human embryonic kidney HEK293 T cells were cultured in Dulbecco's Modified Eagle's Medium with L-Glutamine, $4.5 \mathrm{~g} / \mathrm{L}$ D-Glucose, and $100 \mathrm{mg} / \mathrm{L}$ sodium pyruvate (DMEM; Invitrogen, Carlsbad, CA) supplemented with $10 \%$ fetal bovine serum (FBS), 1X MEM Non-Essential Amino Acids (Invitrogen), and 15 mM HEPES (4-(2-hydroxyethyl)-1piperazineethanesulfonic acid) (Affymetrix, Cleveland, $\mathrm{OH}$ ) at $37^{\circ} \mathrm{C}$ with $5 \% \mathrm{CO}_{2}$.

\section{Immunofluorescence Microscopy and Western Blot}

Cells for immunofluorescence assays were grown and infected on \#1/212 $\times 12-\mathrm{mm}$ glass coverslips for laser-scanning confocal microscopy (LSCM; Electron Microscopy Sciences,

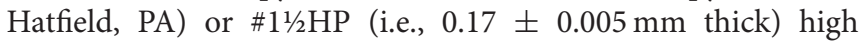
performance glass coverslips (Zeiss, Thornwood, NY) for structured illumination microscopy (SIM). The cells were fixed in $4 \%$ paraformaldehyde (Electron Microscopy Sciences) for $30 \mathrm{~min}$ followed by permeabilization with $0.5 \%$ Triton X-100 for $10 \mathrm{~min}$. Immunofluorescence labeling was performed as previously described (Beyer et al., 2014). For LCSM, coverslips were mounted with Prolong Gold Anti-fade reagent with DAPI (4',6-diamidino-2-phenylindole, Invitrogen) and images were obtained using a Zeiss LSM 700 laser-scanning confocal microscope. Three-dimensional rendering and movies were generated using Volocity Image Analysis Software (PerkinElmer, Waltham, MA). For SIM, coverslips were stained with $1 \mu \mathrm{g} / \mathrm{mL}$ DAPI in 1X PBS for $5 \mathrm{~min}$ and mounted with Prolong Gold Antifade reagent lacking DAPI (Invitrogen). Images were obtained using a Nikon N-SIM super resolution microscope. Lysates of HL-60 cells, host cell-free A. phagocytophilum organisms, or gradient centrifugation fractions were analyzed by SDS-PAGE and Western blot as described (Troese et al., 2011). Primary antibodies used for immunofluorescence and Western blot analyses targeted calreticulin (Sigma-Aldrich, St. Louis, MO), derlin-1 (Sigma-Aldrich and Santa Cruz Biotechnologies [Santa Cruz, CA])), protein disulfide-isomerase (PDI; Sigma-Aldrich), kinectin-1 (Sigma-Aldrich), reticulon-4 (LifeSpan Biosciences Inc, Seattle, WA), A. phagocytophilum APH0032 (Huang et al., 2010b), A. phagocytophilum P44 (Huang et al., 2010b), and A. marginale major surface protein 5 (Msp5) (Visser et al., 1992) (monoclonal antibody clone AnaF16c1, kindly provided by Beverly Hunter and Guy Palmer, Washington State University, Pullman, WA). Alexa Fluor fluorochrome- or horseradish peroxidase-conjugated secondary antibodies were obtained from Invitrogen or Cell Signaling, respectively.

\section{Infection Assays}

HL-60 cells were infected with A. phagocytophilum DC organisms released from infected HL-60 cells by sonication as described (Seidman et al., 2015). ISE6 cells were infected with $A$. phagocytophilum and A. marginale as described for A. phagocytophilum (Huang et al., 2012). RF/6A cells were infected with $A$. phagocytophilum and A. marginale organisms that had been naturally released from infected RF/6A cells into the culture media as follows. Adherent host cells to be infected were seeded onto $\# 1 \frac{1 / 2}{2} 12 \times 12$-mm glass coverslips
(Electron Microscopy Sciences) and overlaid with $200 \mu \mathrm{l}$ of A. phagocytophilum or A. marginale laden media from heavily infected RF/6A cells. Twenty four-well plates containing the coverslips and bacteria laden media were centrifuged at 1000 $g$ for $3 \mathrm{~min}$ to spin the bacteria onto the host cell surfaces followed by a $1 \mathrm{~h}$ incubation at $37^{\circ} \mathrm{C}$ with $5 \% \mathrm{CO}_{2}$. The cells were washed with $1 \mathrm{X}$ PBS to remove unbound bacteria and fresh media was added. The cells were returned to $37^{\circ} \mathrm{C}$ with $5 \%$ $\mathrm{CO}_{2}$ for various time periods, after which they were fixed in $4 \%$ PFA and examined using immunofluorescence microscopy. To assess the effect of ectopic overexpression of mCherry-derlin-1 on the A. phagocytophilum load, HEK-293T cells were transfected with plasmids to enable expression of mCherry-derlin-1 (Nery et al., 2011) (a kind gift from Xandra O. Breakefield [Harvard Medical School, Boston, MA] and Iona A. Armata [Florida State University, Tallahassee, FL]), mCherry (mCherry2-C1 plasmid \#54563; originally from Michael Davidson; Addgene, Cambridge, MA) or mock-transfected as previously described (VieBrock et al., 2014) for $6 \mathrm{~h}$ followed by incubation with host cell-free A. phagocytophilum organisms for 8 or $24 \mathrm{~h}$ prior to processing for LSCM analysis. Alternatively, HEK-293T cells were first infected with $A$. phagocytophilum or mock infected, transfected for $6 \mathrm{~h}$, and processed for LSCM analysis.

\section{Density Gradient Centrifugation}

$2 \times 10^{7}$ uninfected or infected HL-60 cells were washed with icecold 1x PBS twice followed by one wash in cold homogenization buffer (250 mM sucrose, 10 mM Tris- $\mathrm{HCl}, \mathrm{pH}$ 7.4,1 mM EDTA). The cells were suspended in $1 \mathrm{~mL}$ ice-cold homogenization buffer with protease inhibitors (Roche, Indianapolis, IN) and homogenized in a type B dounce homogenizer (Gerresheimer Kimble Chase LLC, Vineland, NJ) for approximately 30 strokes until $\geq 90 \%$ of the host cells were lysed, as verified by the trypan blue exclusion assay. The homogenate was centrifuged at 500 $g$ for $5 \mathrm{~min}$ to remove nuclei and unbroken cells. The postnuclear supernatant was overlaid on a 5, 15,25\% continuous Opti-prep (Sigma-Aldrich) gradient and centrifuged at 200,000 $g$ for $3 \mathrm{~h}$ in an Optima XE-100 ultracentrifuge (Beckman Coulter, Indianapolis, IN). Twelve 1-ml fractions were collected and concentrated by trichloroacetic acid precipitation. Equal volumes of fractions 1-9 were Western blotted and screened with calreticulin and $\mathrm{P} 44$ antibodies.

\section{siRNA Knock Down}

$4 \times 10^{5}$ HEK-293 cells were seeded onto $\# 1 \frac{1}{2} 12 \times 12-\mathrm{mm}$ glass coverslips (Electron Microscopy Sciences). After 16-20 h, 80 ul of 5 uM ON-TARGETplus human Rab10 or derlin-1 siRNA SMARTpool or non-targeting siRNA (GE Dharmacon, Lafayette, CO) was mixed with 320 ul of media and added to the wells. After 72 h, $200 \mathrm{ul}$ of media containing A. phagocytophilum organisms that had been released from infected RF/6A cells was added and the bacteria were spun onto the cells as described above. At 24 or $48 \mathrm{~h}$ post-infection, cells were harvested for Western blot analysis to confirm knockdown, processed for microscopy analyses, or processed for quantitative PCR (QPCR) analyses as described previously (Truchan et al., 2016). Statistical 
significance $(P<0.05)$ was evaluated using the Prism 5.0 software package (Graphpad, San Diego, CA).

\section{RESULTS}

\section{A. phagocytophilum-Occupied Vacuoles (ApVs) Interact with the Host ER}

To investigate if ApVs engage the host ER, A. phagocytophilum infected RF/6A cells were fixed, screened with antibodies specific for the ER lumen markers, calreticulin and PDI (Benham, 2012), and examined by LSCM. Both antibodies revealed a characteristic ER network-like pattern in uninfected and infected cells (Figure 1A). Calreticulin and PDI were considerably enriched in patch-like patterns at the peripheries of ApVs. To determine when ApV-ER association occurs, RF/6A cells were synchronously infected and examined at multiple postinfection time points using antibodies against calreticulin and APH0032, an A. phagocytophilum vacuolar membrane marker that the bacterium expresses predominantly late during the infection cycle (Huang et al., 2010b). As expected (Huang et al., 2010b), APH0032-positive vacuoles were most abundantly detected at 24 and $32 \mathrm{~h}$ (Figure 1B), validating that the infection cycle had proceeded normally. For time points at which APH0032 was not detectable, ApVs were readily visualized due to the presence of DAPI stained bacteria within them. Calreticulin accumulated around all ApVs beginning at $4 \mathrm{~h}$ and remained associated throughout the remainder of the time course (Figures 1B,C). To assess for ApV-ER interactions in another mammalian host cell line, uninfected or A. phagocytophilum infected HL-60 cells were fractionated by a continuous density gradient fractionation method that keeps the ApV intact (Niu et al., 2012). Western blot analysis revealed altered distribution of calreticulin in the fractions of infected vs. uninfected cells, as the ER marker pronouncedly co-migrated to fractions in which the A. phagocytophilum outer membrane protein, P44 (Truchan et al., 2013), was most abundant (Figure 1D). This observation was similar to our previously reported finding that the transGolgi marker, TGN46, but not cis-Golgi marker, GM130, is specifically redistributed to $\mathrm{ApV}$ containing fractions. Together, these data demonstrate that the $\mathrm{ApV}$ associates with the host ER early and maintains this association throughout the infection cycle.

\section{Derlin-1-Positive ER Derived Vesicles are Delivered into the ApV Lumen Where They Associate with A. phagocytophilum Organisms}

Given that two ER lumen markers accumulate around the $\mathrm{ApV}$, it was next assessed if the ER membrane associated protein, derlin-1, exhibited a similar recruitment pattern. Derlin1 is an ER resident protein that is recruited to the ERQC to form part of a membrane-associated complex that directs terminally misfolded proteins to the cytosol for ERAD (Benyair et al., 2015). Derlin-1 displayed a vesicular labeling pattern that, analogous to that observed for calreticulin and PDI, accumulated around $\mathrm{ApV}$ peripheries (Figures 2A,B). Notably, derlin-1 signal was also detected within ApVs in close proximity to DAPI-stained A. phagocytophilum organisms. To more closely examine the possible delivery of derlin-1 positive vesicles into the ApV lumen, a representative APH0032-positive ApV was subjected to $\mathrm{z}$-section image analysis and three-dimensional (3D) rendering. Derlin-1-positive vesicles were observed in close proximity to intravacuolar bacteria within the $\mathrm{ApV}$ throughout the stack of z-section images (Figures 2C,D; Supplementary Movie 1). The timing of derlin-1-positive vesicle localization to and within ApVs was similar to that of calreticulin and PDI immunolabeling of the cytosolic face of the ApV (Figure 1B), as both were modest at $4 \mathrm{~h}$ and considerably more pronounced at all subsequent time points examined (Figures 2E,F).

\section{Derlin-1 Immunolabeling of Intravacuolar A. phagocytophilum Bacteria is Specific and is Reproducible among Different Derlin-1 Antibodies}

To ensure that derlin-1 immunolabeling of intravacuolar A. phagocytophilum organisms was not due to cross-reactivity of the antibody with a bacterial protein, anti-derlin-1 was used to probe Western blotted lysates of uninfected HL-60 cells and host cell-free A. phagocytophilum bacteria. A single band of the expected size for derlin-1 $(22 \mathrm{kDa})$ was detected for the HL-60 cell sample, while no band was detected for the $A$. phagocytophilum sample (Figure 3A). Stripping and reprobing the blot with $B$-actin and P44 antibodies confirmed sample purity. As a complementary approach, derlin-1 expression was knocked down in HEK-293T cells using siRNA. HEK-293T cells were necessary for this purpose because they not only support A. phagocytophilum infection, but also are highly amenable to transfection, whereas RF/6A and HL-60 cells are not (Niu et al., 2012; Beyer et al., 2014; Truchan et al., 2016). HEK293T cells treated with derlin-1-targeting or non-targeting siRNA were infected with A. phagocytophilum followed by Western blot analysis using derlin-1 antibody at $24 \mathrm{~h}$ post-infection. Uninfected HEK-293T cells were included as a control. If derlin1 antibody non-specifically recognized an A. phagocytophilum protein, then knocking down host cell derlin-1 would have no effect on the antibody's ability to recognize the cross-reactive bacterial protein. As observed for HL-60 cells, the antibody detected a single band of the expected size for derlin-1 in all three samples. The band of interest was in considerably lower abundance in the infected, derlin-1 siRNA treated sample (Figure 3B). To verify that the ability to immunolabel $A$. phagocytophilum organisms was not unique to the derlin-1 antibody that had been used for all experiments performed thus far, indirect immunofluorescence analyses of $A$. phagocytophilum infected $\mathrm{RF} / 6 \mathrm{~A}$ cells were repeated to compare this antibody alongside a second derlin- 1 antibody purchased from a different company. The immunolabeling patterns of the host cell ER and intravacuolar bacteria were comparable for both antibodies (Figure 3C). Taken together, these data confirm that derlin-1 antibody labeling of $A$. phagocytophilum organisms within the $\mathrm{ApV}$ is not due to cross-reactivity and is reproducible for two separate derlin-1 antibodies. 

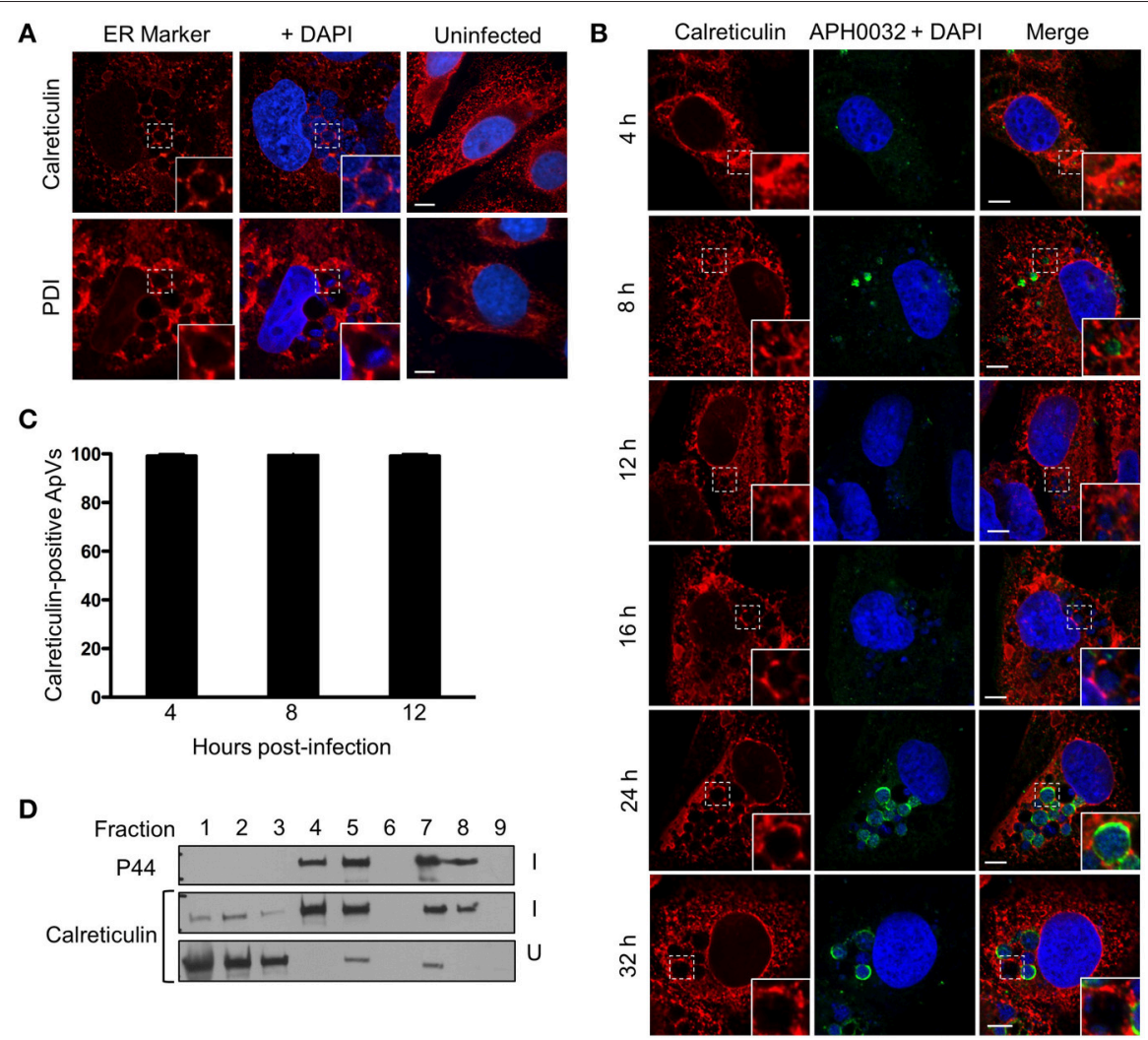

FIGURE 1 | The ApV engages the host ER throughout $\boldsymbol{A}$. phagocytophilum infection of mammalian host cells. (A) ER markers localize to and within the ApV in mammalian cells. A. phagocytophilum infected RF/6A cells that had been screened with antibodies against calreticulin or PDI were visualized using LSCM. (B) The ER is recruited to the ApV early and the association is retained throughout the course of infection. RF/6A cells that had been synchronously infected with

A. phagocytophilum organisms were screened with antibodies against calreticulin and the pathogen derived ApV membrane protein, APH0032, and examined at several post-infection time points using LSCM. (A,B) Host cell nuclei and bacterial DNA were stained with DAPI (blue). The regions that are demarcated by hatched lined boxes indicate the regions magnified in the insets that are demarcated by solid lined boxes. Scale bars, $5 \mu \mathrm{m}$. (C) Percentages of calreticulin-positive ApVs, as identified by DAPI stained intravacuolar $A$. phagocytophilum bacteria, over the course of a synchronous infection. Data are the means and standard deviations for triplicate samples. (D) Calreticulin cofractionates with A. phagocytophilum organisms. Uninfected (U) or A. phagocytophilum-infected HL-60 cells (I) were homogenized and the post-nuclear supernatants were separated by density gradient centrifugation. Successive one-ml fractions were analyzed by Western blot using antibodies against calreticulin and the A. phagocytophilum major surface protein, P44. Results shown are representative of two experiments with similar results.

\section{Ectopically Expressed mCherry-Derlin-1 is Delivered into the ApV, but Inhibits Its Development}

As an additional means of confirming that derlin-1 associates with intravacuolar A. phagocytophilum organisms, HEK-293T cells were transfected to express mCherry-tagged derlin1 or mCherry alone and subsequently infected. At $24 \mathrm{~h}$, mCherry was diffusely distributed throughout the cytosol and nuclei of transfected cells, but not in the ApV lumen (Figure 4A). mCherry-derlin-1 signal exhibited a reticulatelike pattern reminiscent of the ER, as previously described (Nery et al., 2011), and also labeled DAPI-stained intravacuolar A. phagocytophilum bacteria. Notably, however, ApVs were considerably smaller both in diameter and in number in cells expressing mCherryl-derlin-1 as compared to cells expressing only mCherry or mock-infected cells. This result was consistent whether the cells were transfected prior to or after infection. Thus, fluorescently-tagged overexpressed derlin-1 is delivered into the ApV lumen and associates with A. phagocytophilum bacteria. However, $\mathrm{ApV}$ development is pronouncedly hampered in these cells. We rationalized that derlin-1 may become detrimental to A. phagocytophilum at higher levels. We further hypothesized that if derlin-1 negatively influences the bacterium, then knocking down derlin-1 levels would lead to higher A. phagocytophilum loads. Indeed, QPCR analyses of HEK-293T cells that had been treated with derlin-1 or non-targeting siRNA prior to infection confirmed that the A. phagocytophilum load was higher, albeit insignificantly, in cells in which derlin-1 had been knocked down (Figure 4B). This result indirectly suggests that derlin-1 and cellular processes that it regulates may be detrimental to A. phagocytophilum intracellular growth.

\section{A. marginale-Occupied Vacuoles (AmVs) Engage the ER}

To determine if the ability to interface with the ER is conserved in the genus Anaplasma, the above analyses were extended 

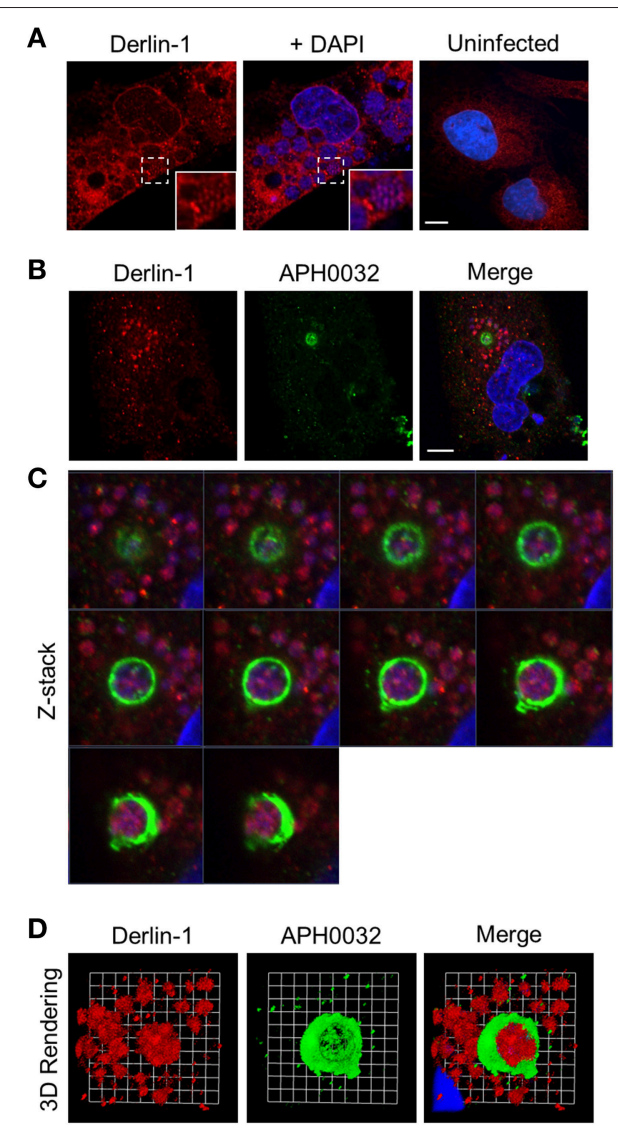

$\mathbf{F}$
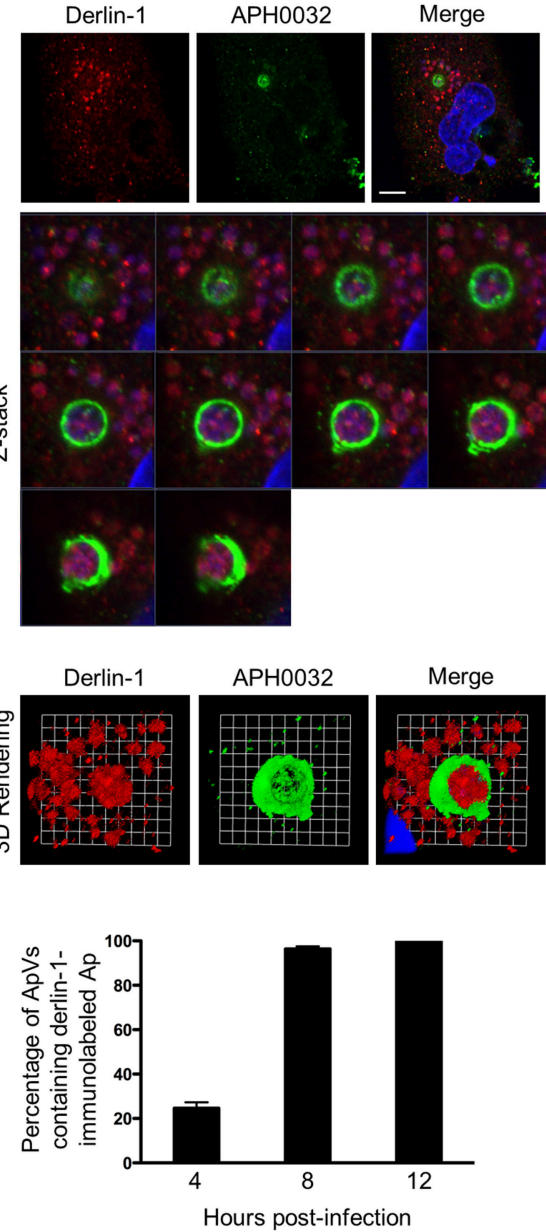

.
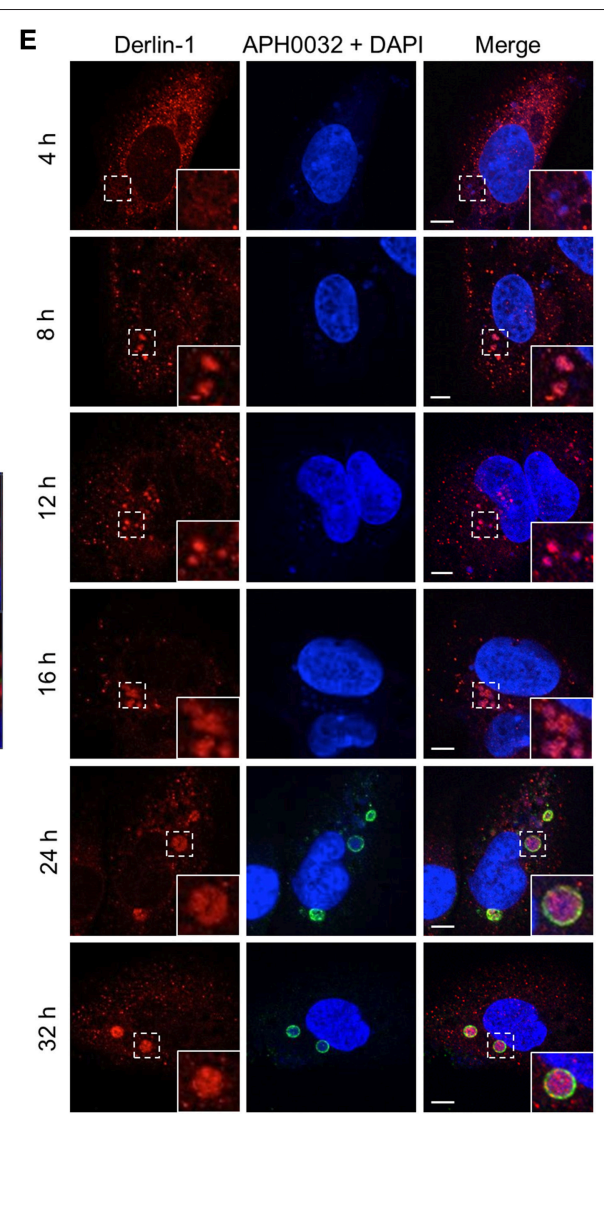
A

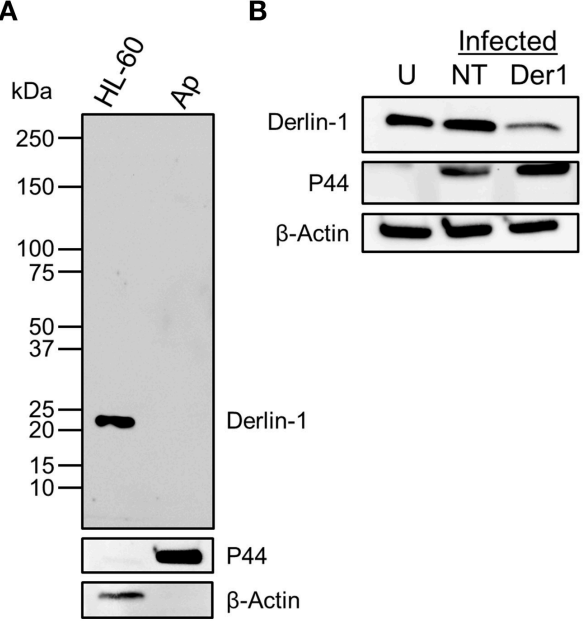

C

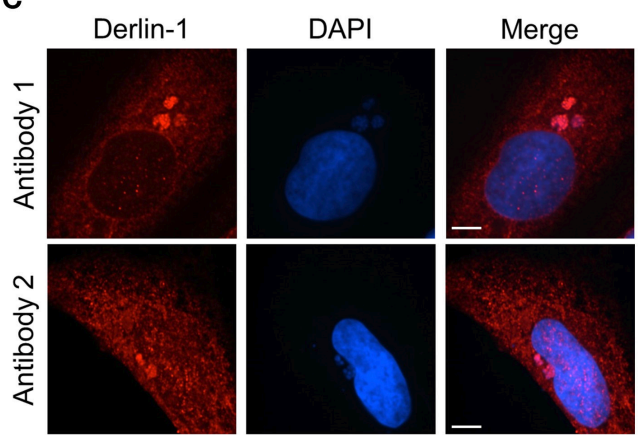

FIGURE 3 | Derlin-1 immunolabeling of intravacuolar $A$. phagocytophilum bacteria is specific and is reproducible among different derlin-1 antibodies. (A,B) Derlin-1 antibody does not cross-react with any $A$. phagocytophilum protein. Western blotted lysates of uninfected $\mathrm{HL}-60$ cells and host cell-free $A$. phagocytophilum (Ap) bacteria (A) or A. phagocytophilum infected derlin-1 siRNA treated (Der1), non-targeting siRNA treated (NT), or uninfected, untreated (U) control HEK-293T cells (B) were probed with antibodies against derlin-1, A. phagocytophilum P44, or B-actin. (C) Two different derlin-1 antibodies produce comparable immunolabeling patterns of the host cell ER and intravacuolar $A$. phagocytophilum organisms. A. phagocytophilum and/or uninfected RF/6A cells were screened with derlin-1 antibodies obtained from two different commercial sources. Scale bars, $5 \mu \mathrm{m}$. Results shown are representative of two experiments with similar results.

\section{Anaplasma spp.-Occupied Vacuoles Interact with Both the RER and SER, and RER Derived Vesicles are Delivered into the ApV Lumen}

Because antibodies specific for markers of both the RER and SER were used in the above analyses, it was unclear if the $\mathrm{ApV}$ and AmV engage one or both of these compartments. Therefore, RF/6A cells infected with either bacterium were screened with antibodies against kinectin-1 or reticulon-4, which are found on the membranes of the RER and SER, respectively (Lynes and Simmen, 2011; Terasaki et al., 2013). Both markers pronouncedly localized in aggregate patterns around ApVs and AmVs (Figure 6). Vesicles positive for kinectin-1, but not reticulon-4 were detected within the ApV in close apposition to DAPI-stained A. phagocytophilum organisms. Neither kinectin1 nor reticulon-4 were detected within the AmV. Thus, the $\mathrm{ApV}$ and $\mathrm{AmV}$ associate with both the SER and RER, but only RER-derived vesicles are translocated into the ApV lumen.

\section{Structured Illumination Microscopy Analyses Confirm That ER Derived Vesicles are Present in Anaplasma spp.-Occupied Vacuoles and Associate with Intravacuolar Bacteria}

LSCM analyses suggested that ER derived vesicles are present within the $\mathrm{ApV}$ and $\mathrm{AmV}$ intravacuolar bacteria. However, because LSCM has a lateral resolution of approximately $200 \mathrm{~nm}$, structures that are separated by less than $200 \mathrm{~nm}$ in the XY plane appear as single fused objects (Allen et al., 2014). Within cells, and presumably within POVs, macro-molecular associations occur in spatial distances of less than $200 \mathrm{~nm}$. To more accurately evaluate if ER derived vesicles associate with intravacuolar Anaplasma spp. organisms, structured illumination microscopy (SIM), a form of super-resolution microscopy that has a lateral resolution of approximately $100 \mathrm{~nm}$ (Allen et al., 2014), was employed. Similar to that observed via LSCM, SIM imaging detected signals for calreticulin and derlin-1 as vesicle-like patterns that accumulated around the peripheries of clusters of DAPI-stained A. phagocytophilum and A. marginale bacteria (Figure 7 ), which were presumably within POVs. Moreover, derlin-1 signal also pronouncedly colocalized with bacteria-associated DAPI signals. These data verify that derlin-1-positive ER derived vesicles are within $100 \mathrm{~nm}$ of the surfaces of $A$. phagocytophilum and $A$. marginale organisms within POVs.

\section{A. phagocytophilum- and \\ A. marginale-Occupied Vacuoles Interact with the ER in Tick Cells}

Because A. marginale and A. phagocytophilum are ticktransmitted pathogens, it was examined if their POVs interact with the ER in ISE6 tick cells. There is a paucity of commercial antibodies against I. scapularis proteins. A BLAST (basic local alignment tool) search revealed that the linear epitopes of human calreticulin and PDI recognized by the commercial antibodies used in this study are present in the I. scapularis orthologs of these proteins (data not shown). Uninfected and A. phagocytophilum- or A. marginale-infected ISE6 cells were subjected to LSCM analyses using these antibodies together with antibody specific for APH0032 (for A. phagocytophilum) or Msp5 (for A. marginale). Both ER markers exhibited the expected perinuclear and network-like pattern in uninfected cells (Figure 8C). In infected cells, calreticulin- and PDI-labeled vesicles were pronouncedly enriched around ApVs and AmVs 


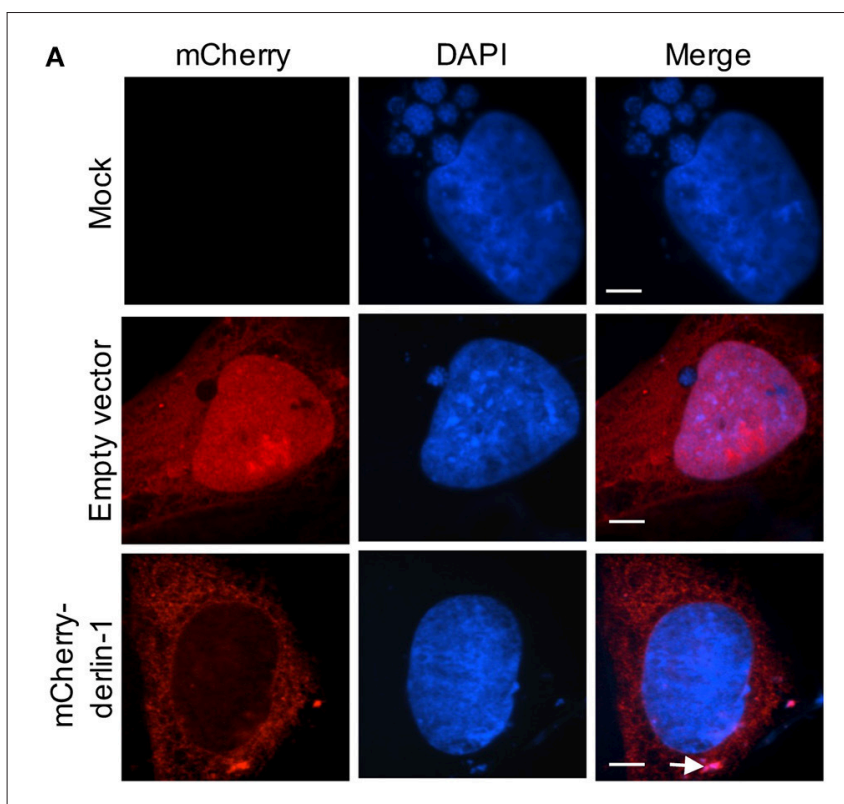

B

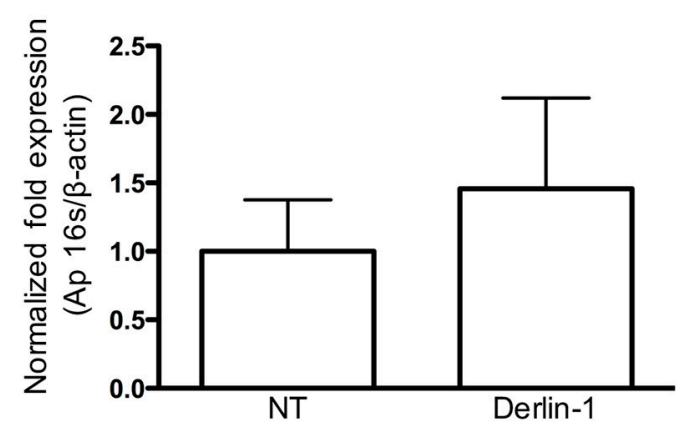

FIGURE 4 | Ectopically overexpressed mCherry-derlin-1 is delivered into and inhibits development of the ApV, and knocking down derlin-1 increases the A. phagocytophilum bacterial load. (A) mCherry-derlin-1 is delivered into the ApV, associated with intravacuolar A. phagocytophilum bacteria, and inhibits ApV development. HEK-293T cells transfected to express mCherry, mCherry-derlin-1, or mock-transfected were incubated with A. phagocytophilum. At $24 \mathrm{~h}$, the cells were fixed, stained with DAPI, and visualized using LSCM. The white arrow in (A) denotes a small mCherry-derlin-1-positive ApV. Scale bars, $0.5 \mu \mathrm{m}$. (B) The A. phagocytophilum load is increased in derlin-1 siRNA-treated cells. HEK-293T cells were treated with derlin-1-targeting or non-targeting (NT) siRNA for $72 \mathrm{~h}$ Following siRNA treatment, the cells were infected with $A$. phagocytophilum for $24 \mathrm{~h}$ and total DNA was isolated and subjected to QPCR analysis.

and also colocalized with Anaplasma organisms within both POV types (Figures 8A,B). Thus, A. phagocytophilum and A. marginale establish interactions with the ER in not only vertebrate, but also invertebrate host cells.

\section{Ultrastructural Analyses of \\ A. phagocytophilum- and \\ A. marginale-Occupied Vacuole Interactions with the ER}

To investigate the interactions of Anaplasma spp-occupied vacuoles with the ER at the ultrastructural level, host cells infected with either species were examined by transmission electron microscopy. As previously reported (Niu et al., 2012), autophagosomes were observed closely apposed to ApVs and autophagic bodies were observed within ApVs in A. phagocytophilum infected HL-60 cells (Figures 9A-C,G,I). ApVs (Figure 9A) and AmVs (Figures 10A-D) were observed in close proximity to ribosome studded RER sheets and the RER formed contacts with and/or wrapped portions of both POV types (Figures 9A-C, 10A-D). Sites where the RER formed contacts on the POV membrane's cytosolic face corresponded with where RCs were closely apposed to the POV membrane's lumenal face (Figures 9A,C, 10A-D). Membrane bound vesicles were commonly observed within $\mathrm{ApV}$ and $\mathrm{AmV}$ lumen in close proximity to bacteria (Figures 9G-I, 10A-C). A. phagocytophilum organisms were occasionally observed wrapped by membranes (Figures 9F,G). The cytosolic faces of ApVs (Figures 9D,E) and AmVs (Figures 10A-D) were often studded with ribosomes. While it could not be ruled out that these were cytosolic ribosomes, the data presented thus far suggested that they were likely derived from the RER and possibly signify ER-Anaplasma spp.-occupied vacuole membrane fusion. Alternatively, the inability to detect a RER sheet in such instances could simply be due to the thin section nature of the sample. In both A. phagocytophilum infected HL-60 cells (Figure 9B) and A. marginale infected ISE6 cells (Figures 10C,D), POVs were commonly observed butted up against the nucleus, phenomena that are potentially related to these pathogens' ER tropism, given that the ER membrane is continuous with the nuclear membrane (Puhka et al., 2012). These data substantiate the association of the ER with $A$. phagocytophilum- and A. marginale-occupied vacuoles, suggest potential fusion of and/or synapse formation between POV and ER membranes, and evidence the presence of vesicles and membranes within POVs in close proximity to bacteria.

\section{Association of the Apv with the ER is Rab10-Independent}

Among the Rab GTPases that A. phagocytophilum selectively recruits to its vacuole are Rab10 and Rab1, both of which are ER associated (Stenmark, 2009; Huang et al., 2010a; English and Voeltz, 2013). Whereas, Rab10 is abundantly detected on ApVs, Rab1 is considerably less so (Huang et al., 2010a). To determine if the association between the $\mathrm{ApV}$ and ER is Rab10-dependent, ER recruitment to the ApV was assessed in HEK-293T cells that had been treated with Rab10 or non-targeting siRNA. Rab10 knockdown was confirmed by Western blot (Figure 11A). Despite multiple attempts, Rab1 could not be knocked down. As previously reported (English and Voeltz, 2013), Rab10-depletion resulted in altered ER morphology. The organelle had lost its network appearance and instead displayed regions of expansive cisternae (Figure 11B). No difference in calreticulin accumulation at the $\mathrm{ApV}$ periphery or the abundant detection of derlin-1 within the ApV lumen was observed between Rab10 knockdown and control cells (Figure 11B). Thus, A. phagocytophilum does not require Rab10 to establish interactions with the ER 


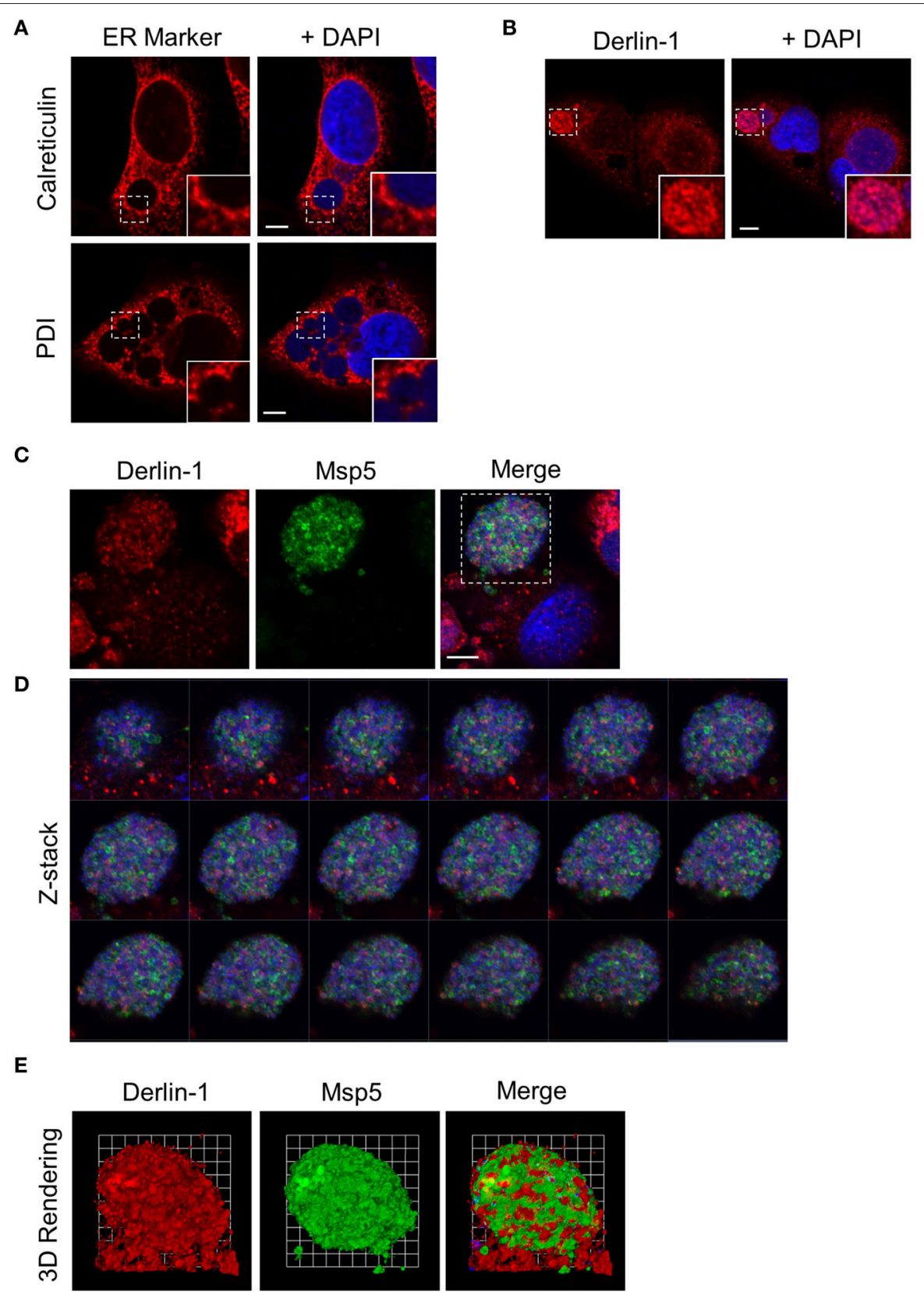

FIGURE 5 | AmVs interact with the ER in mammalian host cells. (A,B) The AmV interacts with the ER and ER-derived vesicles are delivered into its lumen. A. marginale infected RF/6A cells were screened with antibodies against calreticulin (A), PDI (A), or derlin-1 (B). The regions that are demarcated by hatched lined boxes indicate the regions magnified in the insets that are demarcated by solid lined boxes. (C-E) Derlin-1-positive vesicles are present within the AmV in close association with A. marginale bacteria. A. marginale infected RF/6A cells were screened with antibodies against derlin-1 and Msp5 and visualized using LSCM. (D) Z-stack series shows derlin-1-labeled vesicles within the AmV. Successive focal planes of the region in (C) that is demarcated by a hatched line box are presented. (E) 3D rendering of the Z-stack series presented in (D) shows derlin-1-positive vesicles in close proximity to intravacuolar A. marginale organisms. Host cell nuclei and bacteria were stained blue with DAPI. Scale bars, $5 \mu \mathrm{m}$. Results shown are representative of two experiments with similar results.

or to facilitate translocation of ER-derived vesicles into its vacuole.

\section{DISCUSSION}

In this study, we demonstrated that the $\mathrm{ApV}$ and $\mathrm{AmV}$ engage the ER and that ER derived vesicles are delivered into their lumen in mammalian and tick host cells. This strategy is likely important to Anaplasma spp. intracellular survival, as it is initiated in the early hours following the bacterial entry event that generates the POV and continues throughout the entirety of the infection cycle. The delivered vesicles are positive for derlin-1, an ER membrane-associated protein that pronouncedly localizes to the ERQC dynamically and on demand during 

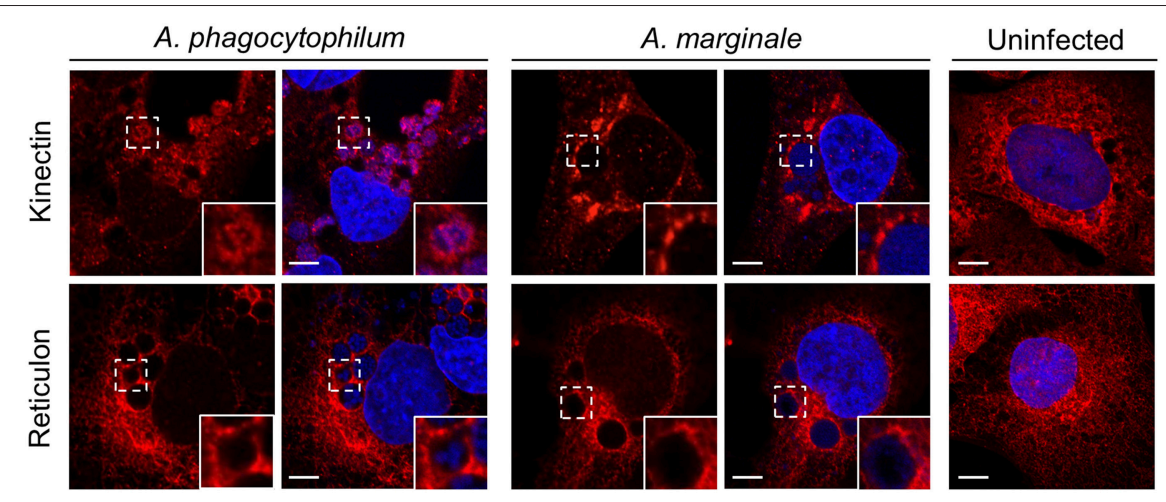

FIGURE 6 | ApVs and AmVs interact with both the RER and SER. Uninfected, A. phagocytophilum infected, or A. marginale infected RF/6A cells were screened with antibodies targeting kinectin-1 or reticulon-4, which are markers for the RER and SER, respectively, and examined using LSCM. Host nuclei and bacterial DNA were stained with DAPI. Scale bars, $5 \mu \mathrm{m}$. Results shown are representative of two experiments with similar results.

\section{A}

A. phagocytophilum

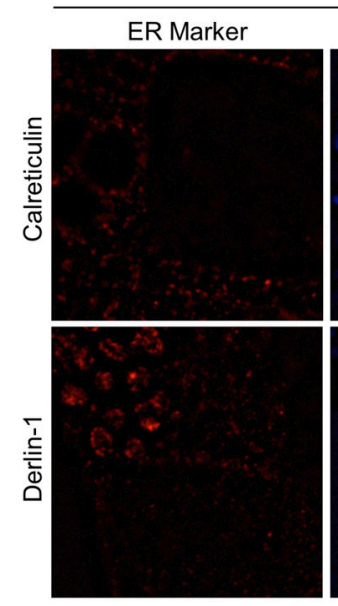

DAPI
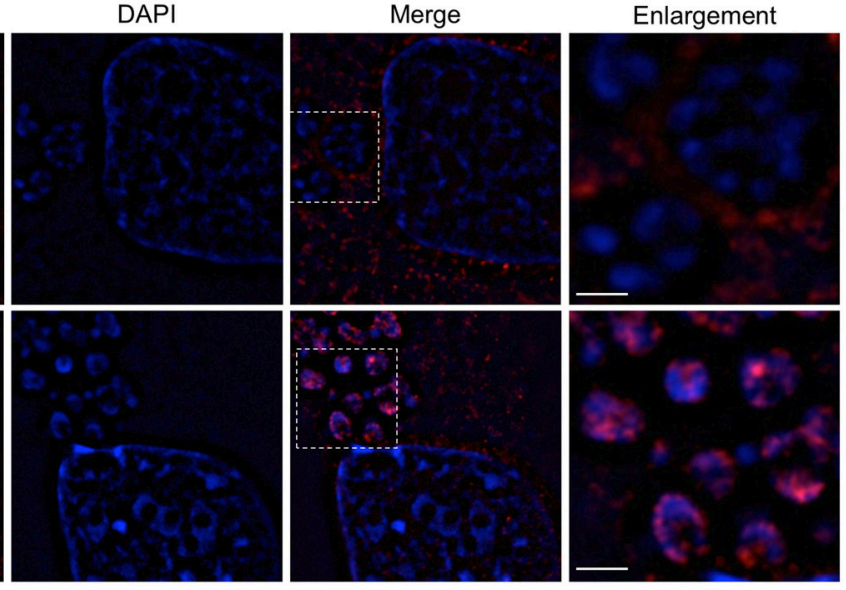

B
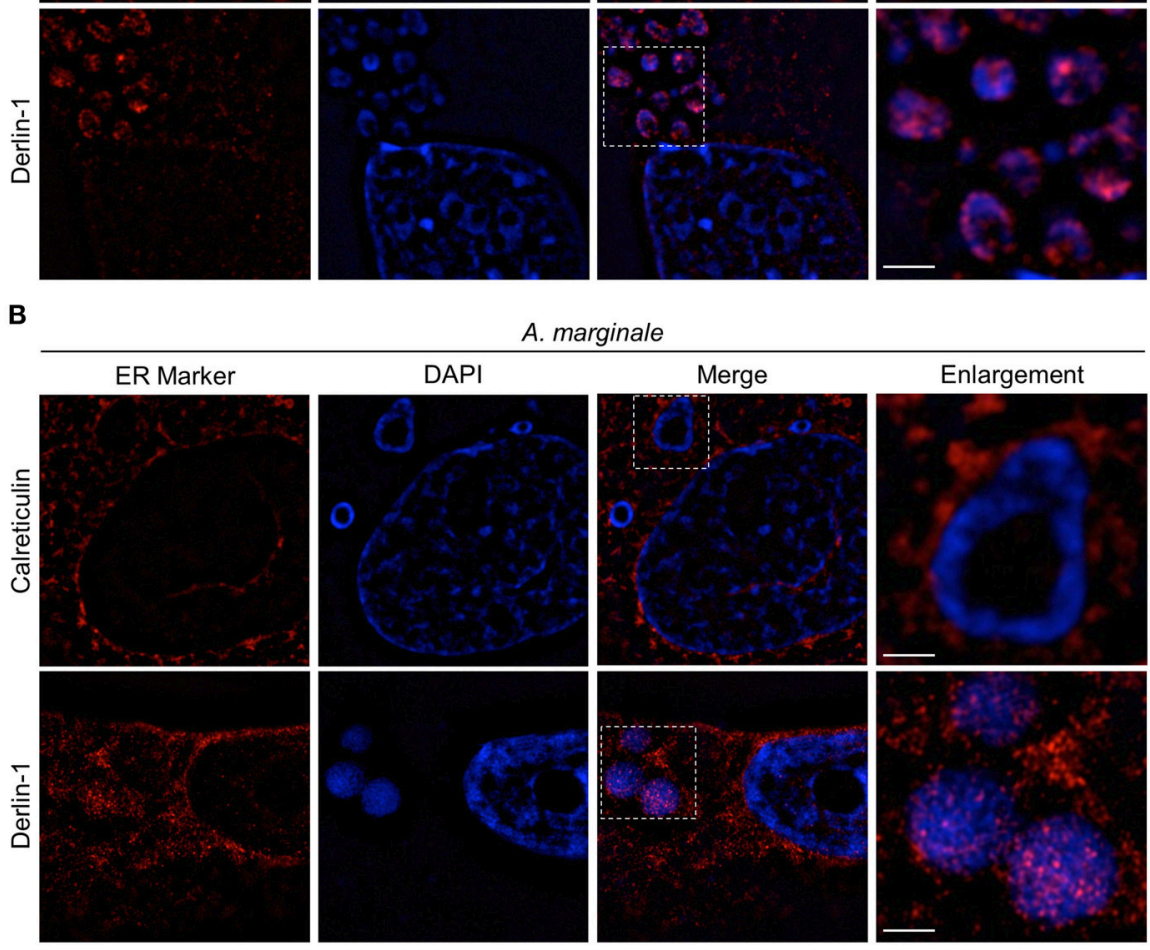

FIGURE 7 | SIM analyses confirm that calreticulin- and derlin-1-positive vesicles are present in the ApV and AmV lumen in close proximity to intravacuolar Anaplasma spp. bacteria. A. phagocytophilum (A) and A. marginale infected RF/6A cells (B) that had been screened with antibodies against calreticulin or derlin-1 were examined by SIM. The regions in the Merge panels that are demarcated by hatched line boxes indicate the regions that are magnified in the Enlargement panels. Host cell nuclei and bacterial DNA were stained with DAPI (blue). Scale bars, $5 \mu \mathrm{m}$. Results shown are representative of two experiments with similar results. 


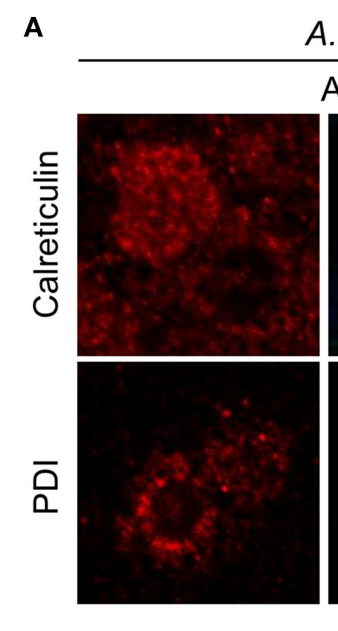

A. phagocytophilum
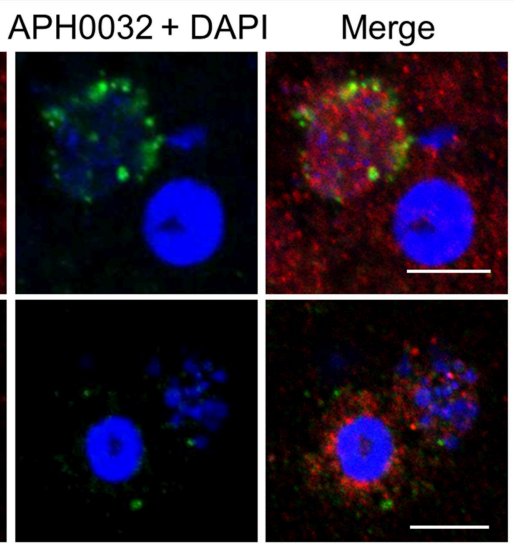

B

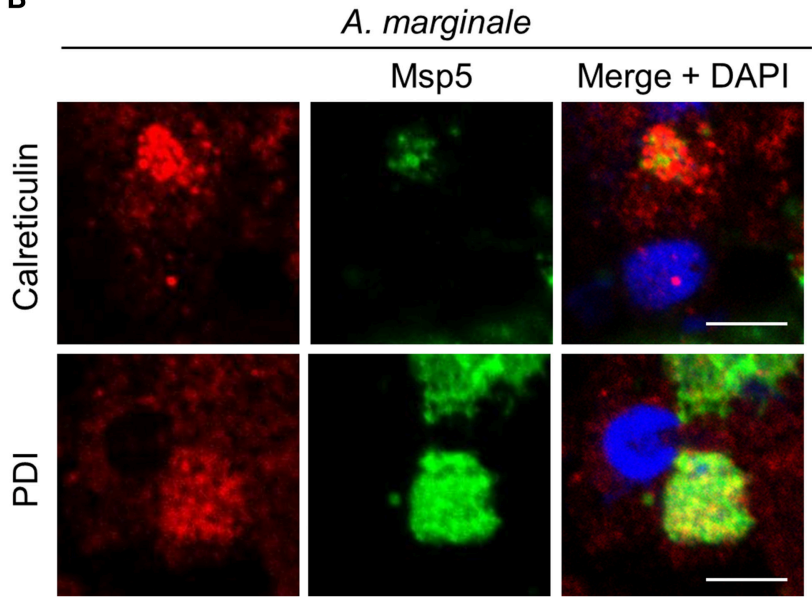

C

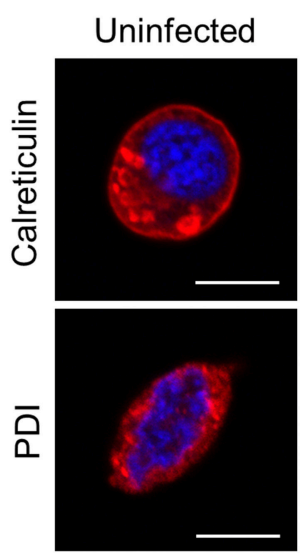

FIGURE 8 | ApVs and AmVs associate with the ER in ISE6 tick cells. Uninfected (C), A. phagocytophilium- (A), or A. marginale-infected ISE6 tick cells (B) were labeled with antibodies against either calreticulin or PDI in combination with APH0032 (for A. phagocytophilum) or Msp2 (for A. marginale) and examined using LSCM. Host nuclei and bacteria were stained blue with DAPI. Scale bars, $5 \mu \mathrm{m}$. Results shown are representative of two experiments with similar results.

ER stress (Lederkremer, 2009; Groisman et al., 2011; Leitman et al., 2014; Benyair et al., 2015). This suggests that the bacteria may selectively hijack membrane traffic from the ERQC. Rab10 is important for vesicular trafficking from the TGN, for ER membrane dynamics, and for maintaining ER morphology, but has not been implicated in ERQC formation or function (Liu and Storrie, 2012; English and Voeltz, 2013). Consistent with this, while Rab10 is essential for A. phagocytophilum TGN parasitism (Truchan et al., 2016), it is dispensable for it to interface with the ER. Thus, the bacterium targets the TGN and ER by distinct mechanisms. Legionella pneumophila modulation of Rab1 is essential for the bacterium to pirate membrane traffic emanating from ERESs (Sherwood and Roy, 2013). Though our inability to knock down Rabl prevented us from discerning if Rab1 is important for A. phagocytophilum to hijack ER membrane traffic, we speculate that it is not, primarily because, like Rab10, Rab1 does not associate with the ERQC (Lederkremer, 2009). Whereas, Anaplasma spp.-occupied vacuoles associate with the RER and SER, RER derived vesicles were detected within lumen of ApVs but not AmVs. Thus, while targeting the ER is a conserved thematic strategy between A. phagocytophilum and A. marginale, species-specific differences exist.

Consistent with immunolabeling of endogenous derlin-1, mCherry-tagged derlin-1 is detected within the ApV lumen and appears to label intravacuolar bacteria. However, ApVs are pronouncedly smaller in both size and number in cells ectopically overexpressing mCherry-derlin-1, but not mCherry. This result suggests that overstimulation of a derlin-1-influenced cellular process may result in aberrantly small ApVs. Derlin1 overexpression has been reported to lead to severe ER stress and ER stress-induced apoptosis (Liang et al., 2014), either or both of which may prevent A. phagocytophilum intracellular growth. A recent study indicated that the translocation of ER associated markers may be exaggerated during chemical fixation and argued that live cell imaging is the gold standard for assessing the significance of such phenomena (Kokes and Valdivia, 2015). Due to the toxic effect of mCherry-derlin-1 

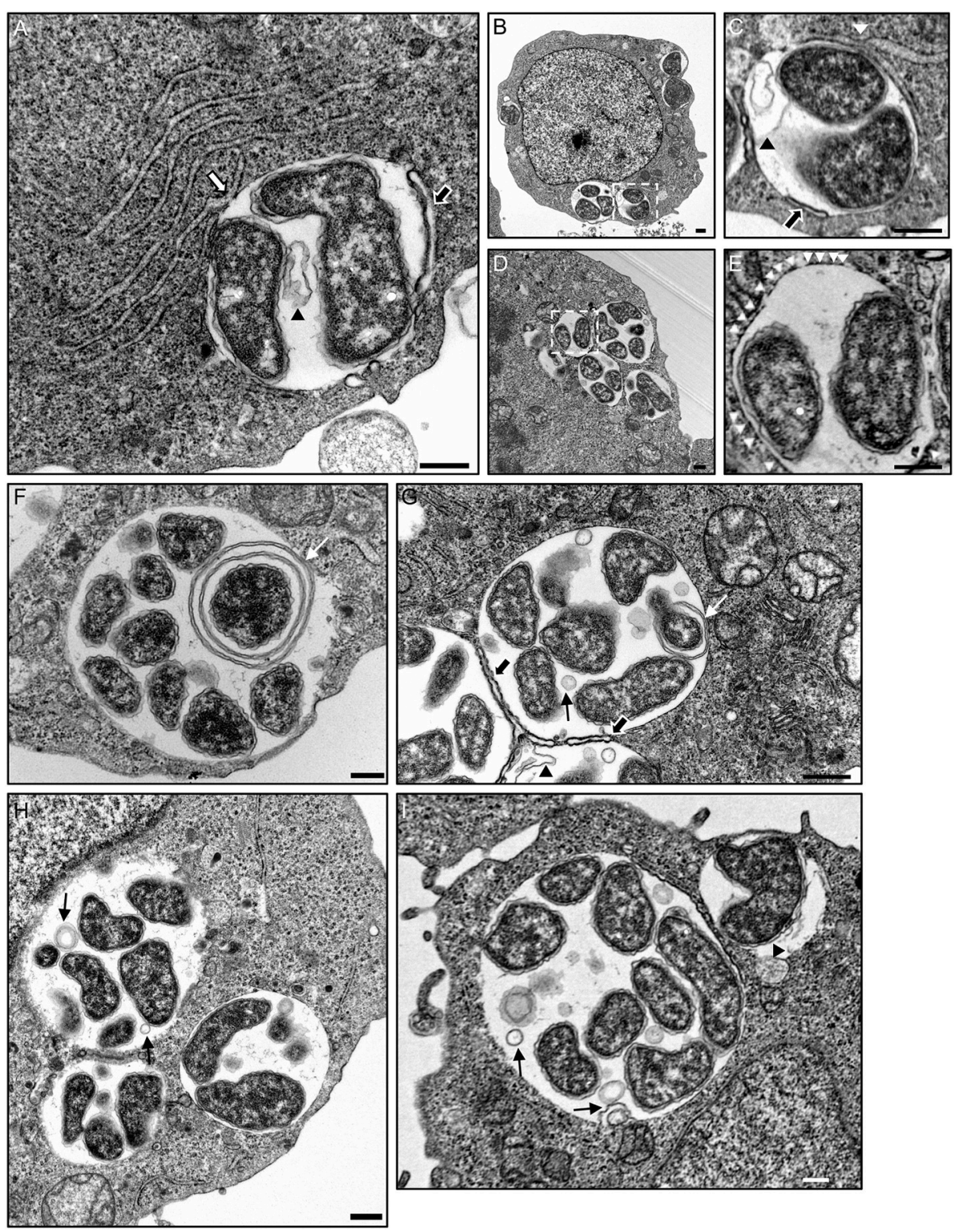

FIGURE 9 | The ApV interacts with the ER as visualized by TEM. A. phagocytophilum infected HL-60 cells were examined by TEM. White hatched boxes denote areas in the images presented in (B,D) that are presented as enlarged panels in (C,E), respectively. The white arrow in (A) denotes a RER-ApV contact site. Black arrows in $\mathbf{( A , C , G )}$ denote autophagosomes contacting the ApV membrane. Black arrowheads in (A,C,G), and (I) demarcate autophagic bodies present within the ApV lumen. White arrowheads in (E) denote ribosomes that label the cytosolic face of the ApV membrane. Thin white arrows in (F,G) demarcate membranes within the ApV lumen associating with $A$. phagocytophilum organisms. Thin black arrows in $\mathbf{( G , H , I ) ~ p o i n t ~ t o ~ v e s i c l e s ~ w i t h i n ~ t h e ~ A p V ~ l u m e n ~ i n ~ c l o s e ~ a p p o s i t i o n ~ t o ~} A$. phagocytophilum bacteria. Scale bars, $0.5 \mu \mathrm{m}$. Results shown are representative of two experiments in which a combined total of over 200 different electron micrographs were analyzed.

overexpression, we could not extend our analyses using live cell imaging. That being said, several lines of indirect evidence collectively argue that derlin-1-positive vesicles are delivered into the ApV lumen to associate with intravacuolar bacteria. First, antibodies specific for derlin-1, but neither calreticulin nor PDI immunolabeled intravacuolar A. phagocytophilum organisms. Second, derlin-1 antibody specifically recognizes its target protein in eukaryotic cells and does not cross-react with an A. phagocytophilum protein. Third, two different derlin-1 antibodies immunolabeled the bacteria. Fourth, mCherry-derlin1 , but not mCherry is detected within the ApV lumen even though both proteins are abundantly present and adjacent to the ApV membrane in transfected cells. Thus, while live cell imaging is a powerful and important technique for assessing the delivery of organelle-derived vesicular traffic into POV lumen and should be employed whenever possible, it is not always going to be a feasible option. In such instances, employing multiple alternative control experiments, as we did 
herein, can circumvent this technical obstacle to reach a valid conclusion.

Anaplasma spp-occupied vacuoles contact the ER and possibly fuse with its membrane at distinct sites. Anaplasma bacteria associate closely on the lumenal sides of such POV-ER membrane contact sites, an observation that is reminiscent of the pathogen synapses formed by chlamydial Type III secretion system apparatuses that connect intravacuolar chlamydiae to the inclusion membrane at ER contact sites (Dumoux et al., 2012). Such chlamydial inclusion membraneER points of contact are also where the bacterial inclusion membrane protein, $\mathrm{IncD}$, recruits host ceramide transfer protein (CERT) that, in turn, interacts with its binding partners and ER membrane resident proteins, VAMP-associated proteinA/B. Recruitment of host sphingomyelin synthases 1 and 2 together with CERT completes a proposed sphingomyelin biosynthetic factory at this host-pathogen interface that may be important in satisfying the chlamydial need for sphingomyelin and may also be a pathogen-orchestrated signaling platform (Derré et al., 2011; Elwell et al., 2011; Agaisse and Derre, 2014). Given that we recently demonstrated that host cell-free A. phagocytophilum DCs are enriched in multiple ceramide and sphingomyelin sub-species (Truchan et al., 2016), moving forward it will be important to discern whether Anaplasma spp.-occupied vacuoles have similar metabolic/signaling platforms at their ER-POV membrane contact sites, the bacterial factors responsible for facilitating their formation, and their contributions to Anaplasma spp. pathobiology.

Though other intracellular bacterial pathogens have been shown to replicate within ER-derived compartments (Swanson and Isberg, 1995; Roy et al., 2006; Dumoux et al., 2012; Celli and Tsolis, 2015), the concept of intact ER vesicles being delivered into the POV is fairly novel. Prior to this study, the chlamydial inclusion was the only POV demonstrated to intimately contact the ER and ingest intact ER derived vesicles into its lumen (Dumoux et al., 2012). The ApV and the chlamydial inclusion each also associates with the Golgi apparatus and selectively hijacks its exocytosed vesicles, which, like ER vesicles, are translocated into the POV lumen to associate with intravacuolar bacteria (Heuer et al., 2009; Capmany and Damiani, 2010; Moore et al., 2011; Pokrovskaya et al., 2012; AlZeer et al., 2014; Dille et al., 2014; Truchan et al., 2016). Hijacking ER- and Golgi-derived traffic is essential for chlamydiae to convert from the replicative to infectious form (Heuer et al., 2009; Dumoux et al., 2012). TGN parasitism is critical for A. phagocytophilum to complete its biphasic developmental cycle (Dumoux et al., 2012; Truchan et al., 2016). Whether pirating a derlin-1-positive ER sub-compartment contributes to Anaplasma spp. RC-to-DC conversion is unknown, as is the pathobiological benefit that hijacking this specific ER domain affords. Regardless, data presented here and in our previous study demonstrate the importance of hijacking multiple secretory organelles to Anaplasma spp. infection. Moreover, this study adds to a growing body of literature that demonstrates that pathogens in the families Anaplasmataceae and Chlamydiaceae, while evolutionarily distinct in terms of whether or not
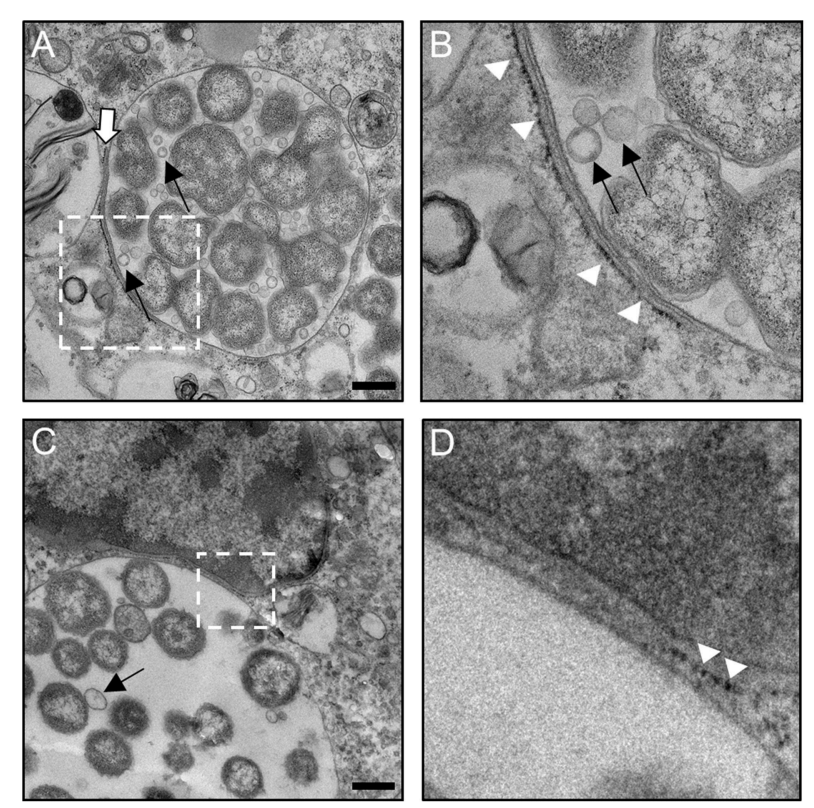

FIGURE 10 | The AmV interacts with the ER as visualized by TEM. $A$. marginale infected ISE6 cells were examined by TEM. White hatched boxes denote areas in the images presented in $(\mathbf{A}, \mathbf{C})$ that are presented as enlarged panels in (B,D), respectively. The white arrow in $(\mathbf{A})$ denotes a RER-AmV contact site. White arrowheads in (B,D) point to ribosomes that label the cytosolic face of the AmV membrane. Thin black arrows in (A-C) demarcate vesicles within the AmV lumen in close apposition to $A$. marginale organisms. Scale bars, $0.5 \mu \mathrm{m}$. Results shown are representative of two experiments in which a combined total of over 40 different electron micrographs were analyzed.

they are vector-transmitted and the host cell types that they infect, exhibit a conserved demand for parasitizing the secretory pathway and targeting secretory organelle derived vesicles into their POVs (Beatty, 2006; Cocchiaro et al., 2008; Capmany and Damiani, 2010; Dumoux et al., 2012; Boncompain et al., 2014; Truchan et al., 2016). While the responsible mechanism(s) for such membrane fusion-independent delivery is undefined, it was recently demonstrated that two Toxoplasma gondii encoded proteins that localize to the parasitophorous vacuole membrane form a conduit that facilitates passage of small molecules between the vacuole and host cytosol (Gold et al., 2015). Perhaps functionally analogous proteins exist on bacterial POVs to import intact host cell derived vesicles.

What possible benefits could hijacking ER traffic afford A. phagocytophilum and A. marginale? As the pathogens are auxotrophic for most amino acids (Brayton et al., 2005; Rikihisa, 2011), ER vesicles transported into their vacuoles could provide proteins that get degraded and used as an amino acid source, as has been demonstrated for autophagic bodies that are delivered into the $\mathrm{ApV}$ as a result of $\mathrm{ApV}$-autophagosome fusion (Niu et al., 2012). Anaplasma spp. could use the amino acids for protein synthesis or as a source of carbon, nitrogen, and energy by feeding the amino acids into the Kreb's cycle. The latter strategy has been well-documented for L. pneumophila (Pine 

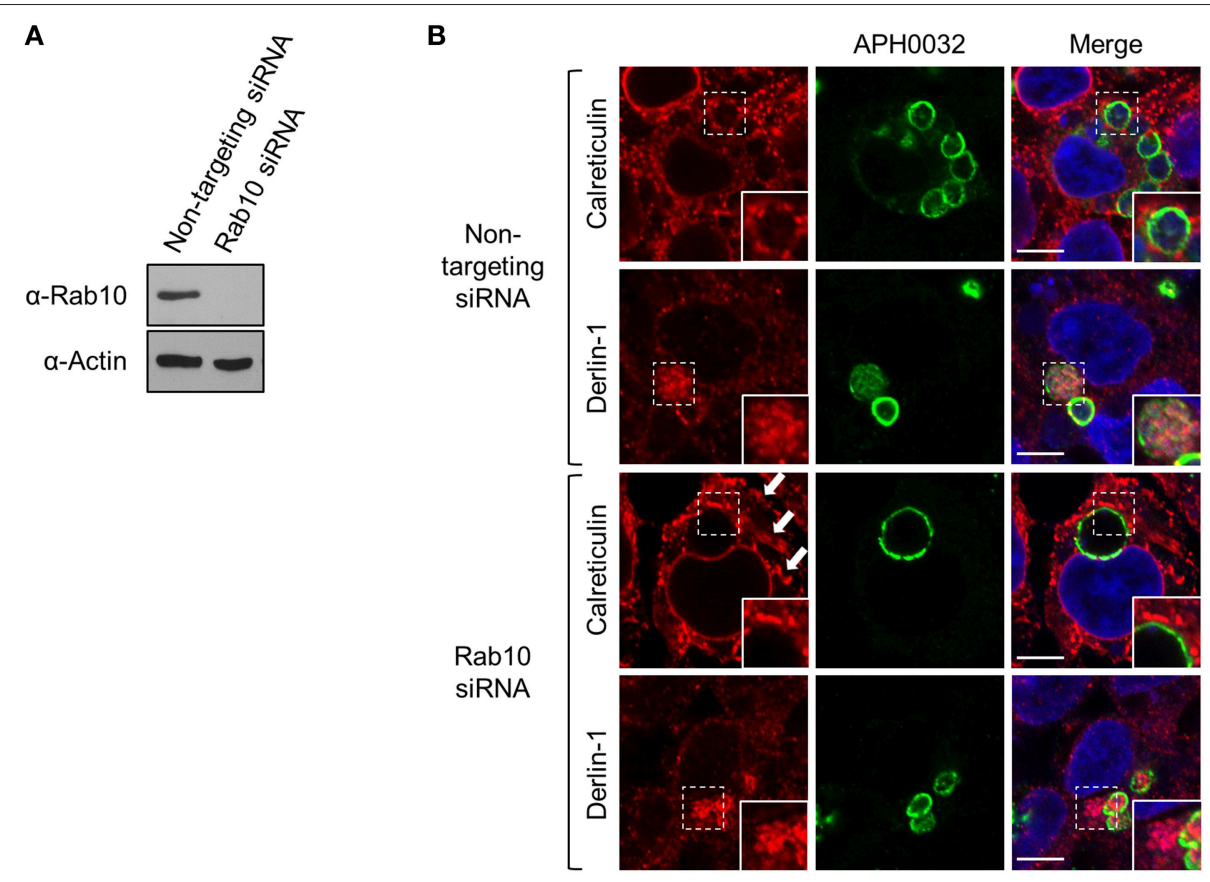

FIGURE 11 | ApV association with the ER is Rab10-independent. HEK-293T cells were treated with Rab10-targeting or non-targeting siRNA for $72 \mathrm{~h}$. (A) Lysates of non-targeting or Rab10 siRNA treated cells were examined by Western blot for Rab10 knockdown. (B) Following siRNA treatment, the cells were infected with A. phagocytophilum for $48 \mathrm{~h}$, fixed, screened with antibodies against APH0032 and calreticulin or derlin-1, and examined using LSCM. Host cell nuclei and bacterial DNA were stained with DAPI. Regions demarcated by hatched line boxes are magnified in the corresponding inset images that are denoted by a solid line boxes. Arrows point to regions of expansive cisternae, which is characteristic ER morphology in Rab10-depleted cells. Scale bars, $5 \mu \mathrm{m}$. Results shown are representative of two experiments with similar results.

et al., 1979; Tesh et al., 1983; Price et al., 2014). Autophagosomes are believed to be formed from ER membrane and it was recently found that $70 \%$ of autophagosomes contain portions of the ER (Hayashi-Nishino et al., 2009; Shibutani and Yoshimori, 2014). However, autophagosomes do not contain ribosomes, which were detected on or in close apposition to the cytosolic faces of ApVs and AmVs, or calreticulin and PDI (Dunn, 1994; HayashiNishino et al., 2009; Lamb et al., 2013; Shibutani and Yoshimori, 2014), which heavily label Anaplasma spp.-occupied vacuoles. Thus, while the ApV engages autophagosomes (Niu et al., 2012), both it and the AmV establish separate interactions with the ER.

The recently penned term, nutritional virulence, refers to the condition that without access to essential nutrients, pathogens cannot survive to cause disease (Abu Kwaik and Bumann, 2013). ER-POV interactions may help satisfy the nutritional virulence requirements of $A$. phagocytophilum during infection of leukocytes and of both it and A. marginale during infection of endothelial and tick cells. It will be important to verify which specific ER subdomain(s) Anaplasma spp. target, the relevance of this strategy to their infection cycles, and the molecular mechanisms by which they are orchestrated. Such knowledge could potentially be exploited as a novel means for treating human or animal infections caused by Anaplasma spp. or for eliminating the pathogens from their tick vectors.

\section{AUTHOR CONTRIBUTIONS}

JC, HT, SN designed experiments and analyzed the data. HT, CC, $\mathrm{KH}$, FM performed the experiments and analyzed the data. JC and HT wrote the paper.

\section{FUNDING}

This study was supported by funding from National Institutes of Health Grants R01 AI072683, National Center for Advancing Translational Sciences Grant UL1TR000058, and the Center for Clinical and Translational Research Endowment Fund of VCU (to JC). LSCM, SIM, and electron microscopy were performed at the VCU Microscopy Facility, which is supported in part with funding from NIH-NINDS Center core grant 5P30NS047463 and NIH-NCI Cancer Center Support Grant (P30 CA016059). SN: U. S. Department of Agriculture-Agricultural Research Service Project \#534832000-033-00D and National Institutes of Health R37 AI44005.

\section{ACKNOWLEDGMENTS}

We thank Xandra O. Breakefield (Harvard Medical School, Boston, MA) and Iona A. Armata (Florida State University, Tallahassee, FL) for the mCherry-derlin-1 construct, Yasuko 
Rikihisa and Qingming Xiong (Ohio State University, Columbus, $\mathrm{OH}$ ) for helpful advice on density gradient purification of ApVs, Beverly Hunter and Guy Palmer (Washington State University, Pullman, WA) for providing monoclonal antibody AnaF16C1, Gerardo Lederkremer (Tel Aviv University) and Kyle Rodino (Virginia Commonwealth University [VCU]) for valuable discussions, and Gretchen Diffendall (VCU) for technical assistance. We thank Dr. Christine Davitt at the

\section{REFERENCES}

Abu Kwaik, Y., and Bumann, D. (2013). Microbial quest for food in vivo: "nutritional virulence" as an emerging paradigm. Cell. Microbiol. 15, 882-890. doi: $10.1111 / \mathrm{cmi} .12138$

Agaisse, H., and Derre, I. (2014). Expression of the effector protein IncD in Chlamydia trachomatis mediates recruitment of the lipid transfer protein CERT and the endoplasmic reticulum-resident protein VAPB to the inclusion membrane. Infect. Immun. 82, 2037-2047. doi: 10.1128/IAI.01530-14

Allen, J. R., Ross, S. T., and Davidson, M. W. (2014). Structured illumination microscopy for superresolution. Chemphyschem 15, 566-576. doi: 10.1002/cphc.201301086

Al-Zeer, M. A., Al-Younes, H. M., Kerr, M., Abu-Lubad, M., Gonzalez, E., Brinkmann, V., et al. (2014). Chlamydia trachomatis remodels stable microtubules to coordinate Golgi stack recruitment to the chlamydial inclusion surface. Mol. Microbiol. 94, 1285-1297. doi: 10.1111/mmi.12829

Beatty, W. L. (2006). Trafficking from CD63-positive late endocytic multivesicular bodies is essential for intracellular development of Chlamydia trachomatis. J. Cell Sci. 119, 350-359. doi: $10.1242 /$ jcs. 02733

Benham, A. M. (2012). Protein secretion and the endoplasmic reticulum. Cold Spring Harb. Perspect. Biol. 4:a012872. doi: 10.1101/cshperspect.a012872

Benyair, R., Ogen-Shtern, N., and Lederkremer, G. Z. (2015). Glycan regulation of ER-associated degradation through compartmentalization. Semin. Cell Dev. Biol. 41, 99-109. doi: 10.1016/j.semcdb.2014.11.006

Beyer, A. R., Truchan, H. K., May, L. J., Walker, N. J., Borjesson, D. L., and Carlyon, J. A. (2014). The Anaplasma phagocytophilum effector AmpA hijacks host cell SUMOylation. Cell Microbiol. 17, 504-519. doi: 10.1111/cmi.12380

Boncompain, G., Muller, C., Meas-Yedid, V., Schmitt-Kopplin, P., Lazarow, P. B., and Subtil, A. (2014). The intracellular bacteria Chlamydia hijack peroxisomes and utilize their enzymatic capacity to produce bacteria-specific phospholipids. PLoS ONE 9:e86196. doi: 10.1371/journal.pone.0086196

Brayton, K. A., Kappmeyer, L. S., Herndon, D. R., Dark, M. J., Tibbals, D. L., Palmer, G. H., et al. (2005). Complete genome sequencing of Anaplasma marginale reveals that the surface is skewed to two superfamilies of outer membrane proteins. Proc. Natl. Acad. Sci. U.S.A. 102, 844-849. doi: 10.1073/pnas.0406656102

Brumell, J. H., and Scidmore, M. A. (2007). Manipulation of rab GTPase function by intracellular bacterial pathogens. Microbiol. Mol. Biol. Rev. 71, 636-652. doi: 10.1128/MMBR.00023-07

Capmany, A., and Damiani, M. T. (2010). Chlamydia trachomatis intercepts Golgi-derived sphingolipids through a Rab14-mediated transport required for bacterial development and replication. PLOS ONE 5:e14084. doi: 10.1371/journal.pone.0014084

Carlyon, J. A. (2012). "Establishing intracellular infection: modulation of host cell functions (Anaplasmataceae)," in Intracellular Pathogens II: Rickettsiales, eds G. H. Palmer and A. Azad (Washington, DC: ASM Press), 3A.2.1-3A.2.30.

CDC (2013). Notice to readers: final 2012 reports of nationally notifiable infectious diseases. MMWR Morb. Mortal. Wkly. Rep. 62, 669-682.

Celli, J., and Tsolis, R. M. (2015). Bacteria, the endoplasmic reticulum and the unfolded protein response: friends or foes? Nat. Rev. Microbiol. 13, 71-82. doi: $10.1038 /$ nrmicro3393

Cocchiaro, J. L., Kumar, Y., Fischer, E. R., Hackstadt, T., and Valdivia, R. H. (2008). Cytoplasmic lipid droplets are translocated into the lumen of the Chlamydia trachomatis parasitophorous vacuole. Proc. Natl. Acad. Sci. U.S.A. 105, 9379-9384. doi: 10.1073/pnas.0712241105

Derré, I., Swiss, R., and Agaisse, H. (2011). The lipid transfer protein CERT interacts with the Chlamydia inclusion protein $\mathrm{IncD}$ and participates to
Franceschi Microscopy and Imaging Center (Washington State University, Pullman, WA) for technical assistance.

\section{SUPPLEMENTARY MATERIAL}

The Supplementary Material for this article can be found online at: http://journal.frontiersin.org/article/10.3389/fcimb. 2016.00022

ER-Chlamydia inclusion membrane contact sites. PLoS Pathog. 7:e1002092. doi: 10.1371/journal.ppat.1002092

Dille, S., Herbst, K., Volceanov, L., Nolke, T., Kretz, O., and Hacker, G. (2014). Golgi fragmentation and sphingomyelin transport to Chlamydia trachomatis during penicillin-induced persistence do not depend on the cytosolic presence of the chlamydial protease CPAF. PLoS ONE 9:e103220. doi: 10.1371/journal.pone. 0103220

Dumoux, M., Clare, D. K., Saibil, H. R., and Hayward, R. D. (2012). Chlamydiae assemble a pathogen synapse to hijack the host endoplasmic reticulum. Traffic 13, 1612-1627. doi: 10.1111/tra.12002

Dunn, W. A. Jr. (1994). Autophagy and related mechanisms of lysosomemediated protein degradation. Trends Cell Biol. 4, 139-143. doi: 10.1016/09628924(94)90069-8

Elwell, C. A., Jiang, S., Kim, J. H., Lee, A., Wittmann, T., Hanada, K., et al. (2011). Chlamydia trachomatis co-opts GBF1 and CERT to acquire host sphingomyelin for distinct roles during intracellular development. PLoS Pathog. 7:e1002198. doi: 10.1371/journal.ppat.1002198

English, A. R., and Voeltz, G. K. (2013). Rab10 GTPase regulates ER dynamics and morphology. Nat. Cell Biol. 15, 169-178. doi: 10.1038/ncb2647

Gold, D. A., Kaplan, A. D., Lis, A., Bett, G. C., Rosowski, E. E., Cirelli, K. M., et al. (2015). The toxoplasma dense granule proteins GRA17 and GRA23 mediate the movement of small molecules between the host and the Parasitophorous vacuole. Cell Host Microbe 17, 642-652. doi: 10.1016/j.chom.2015.04.003

Goodman, J. L., Nelson, C., Vitale, B., Madigan, J. E., Dumler, J. S., Kurtti, T. J., et al. (1996). Direct cultivation of the causative agent of human granulocytic ehrlichiosis. N. Engl. J. Med. 334, 209-215. doi: 10.1056/NEJM1996012533 40401

Groisman, B., Shenkman, M., Ron, E., and Lederkremer, G. Z. (2011). Mannose trimming is required for delivery of a glycoprotein from EDEM1 to XTP3-B and to late endoplasmic reticulum-associated degradation steps. J. Biol. Chem. 286, 1292-1300. doi: 10.1074/jbc.M110.154849

Hayashi-Nishino, M., Fujita, N., Noda, T., Yamaguchi, A., Yoshimori, T., and Yamamoto, A. (2009). A subdomain of the endoplasmic reticulum forms a cradle for autophagosome formation. Nat. Cell Biol. 11, 1433-1437. doi: $10.1038 /$ ncb 1991

Herron, M. J., Ericson, M. E., Kurtti, T. J., and Munderloh, U. G. (2005). The interactions of Anaplasma phagocytophilum, endothelial cells, and human neutrophils. Ann. N.Y. Acad. Sci. 1063, 374-382. doi: 10.1196/annals. 1355.090

Heuer, D., Rejman Lipinski, A., Machuy, N., Karlas, A., Wehrens, A., Siedler, F., et al. (2009). Chlamydia causes fragmentation of the Golgi compartment to ensure reproduction. Nature 457, 731-735. doi: 10.1038/nature07578

Huang, B., Hubber, A., McDonough, J. A., Roy, C. R., Scidmore, M. A., and Carlyon, J. A. (2010a). The Anaplasma phagocytophilum-occupied vacuole selectively recruits Rab-GTPases that are predominantly associated with recycling endosomes. Cell. Microbiol. 12, 1292-1307. doi: 10.1111/j.14625822.2010.01468.x

Huang, B., Ojogun, N., Ragland, S. A., and Carlyon, J. A. (2012). Monoubiquitinated proteins decorate the Anaplasma phagocytophilumoccupied vacuolar membrane. FEMS Immunol. Med. Microbiol. 64, 32-41. doi: 10.1111/j.1574-695X.2011.00873.x

Huang, B., Troese, M. J., Howe, D., Ye, S., Sims, J. T., Heinzen, R. A., et al. (2010b). Anaplasma phagocytophilum APH_0032 is expressed late during infection and localizes to the pathogen-occupied vacuolar membrane. Microb. Pathog. 49, 273-284. doi: 10.1016/j.micpath.2010.06.009

Kocan, K. M., de la Fuente, J., Blouin, E. F., Coetzee, J. F., and Ewing, S. A. (2010). The natural history of Anaplasma marginale. Vet. Parasitol. 167, 95-107. doi: 10.1016/j.vetpar.2009.09.012 
Kocan, K. M., de la Fuente, J., Guglielmone, A. A., and Melendez, R. D. (2003). Antigens and alternatives for control of Anaplasma marginale infection in cattle. Clin. Microbiol. Rev. 16, 698-712. doi: 10.1128/CMR.16.4.698712.2003

Kokes, M., and Valdivia, R. H. (2015). Differential translocation of host cellular materials into the Chlamydia trachomatis inclusion lumen during chemical fixation. PLoS ONE 10:e0139153. doi: 10.1371/journal.pone.0139153

Lamb, C. A., Yoshimori, T., and Tooze, S. A. (2013). The autophagosome: origins unknown, biogenesis complex. Nat. Rev. Mol. Cell Biol. 14, 759-774. doi: $10.1038 / \mathrm{nrm} 3696$

Lederkremer, G. Z. (2009). Glycoprotein folding, quality control and ERassociated degradation. Curr. Opin. Struct. Biol. 19, 515-523. doi: 10.1016/j.sbi.2009.06.004

Leitman, J., Shenkman, M., Gofman, Y., Shtern, N. O., Ben-Tal, N., Hendershot, L. M., et al. (2014). Herp coordinates compartmentalization and recruitment of HRD1 and misfolded proteins for ERAD. Mol. Biol. Cell 25, 1050-1060. doi: 10.1091/mbc.E13-06-0350

Liang, C. J., Chang, Y. C., Chang, H. C., Wang, C. K., Hung, Y. C., Lin, Y. E., et al. (2014). Derlin-1 regulates mutant VCP-linked pathogenesis and endoplasmic reticulum stress-induced apoptosis. PLoS Genet. 10:e1004675. doi: 10.1371/journal.pgen.1004675

Liu, S., and Storrie, B. (2012). Are Rab proteins the link between Golgi organization and membrane trafficking? Cell. Mol. Life Sci. 69, 4093-4106. doi: 10.1007/s00018-012-1021-6

Lynes, E. M., and Simmen, T. (2011). Urban planning of the endoplasmic reticulum (ER): how diverse mechanisms segregate the many functions of the ER. Biochim. Biophys. Acta 1813, 1893-1905. doi: 10.1016/j.bbamcr.2011.06.011

Moore, E. R., Mead, D. J., Dooley, C. A., Sager, J., and Hackstadt, T. (2011). The trans-Golgi SNARE syntaxin 6 is recruited to the chlamydial inclusion membrane. Microbiology 157, 830-838. doi: 10.1099/mic.0.045856-0

Munderloh, U. G., Lynch, M. J., Herron, M. J., Palmer, A. T., Kurtti, T. J., Nelson, R. D., et al. (2004). Infection of endothelial cells with Anaplasma marginale and A. phagocytophilum. Vet. Microbiol. 101, 53-64. doi: 10.1016/j.vetmic.2004.02.011

Nery, F. C., Armata, I. A., Farley, J. E., Cho, J. A., Yaqub, U., Chen, P., et al. (2011). Torsin A participates in endoplasmic reticulum-associated degradation. Nat. Commun. 2, 393. doi: 10.1038/ncomms1383

Niu, H., Xiong, Q., Yamamoto, A., Hayashi-Nishino, M., and Rikihisa, Y. (2012). Autophagosomes induced by a bacterial Beclin 1 binding protein facilitate obligatory intracellular infection. Proc. Natl. Acad. Sci. U.S.A. 109, 20800-20807. doi: 10.1073/pnas.1218674109

Pine, L., George, J. R., Reeves, M. W., and Harrell, W. K. (1979). Development of a chemically defined liquid medium for growth of Legionella pneumophila. J. Clin. Microbiol. 9, 615-626.

Pokrovskaya, I. D., Szwedo, J. W., Goodwin, A., Lupashina, T. V., Nagarajan, U. M., and Lupashin, V. V. (2012). Chlamydia trachomatis hijacks intra-Golgi COG complex-dependent vesicle trafficking pathway. Cell. Microbiol. 14, 656-668. doi: 10.1111/j.1462-5822.2012.01747.x

Price, C. T., Richards, A. M., and Abu Kwaik, Y. (2014). Nutrient generation and retrieval from the host cell cytosol by intra-vacuolar Legionella pneumophila. Front. Cell. Infect. Microbiol. 4:111. doi: 10.3389/fcimb.2014.00111

Puhka, M., Joensuu, M., Vihinen, H., Belevich, I., and Jokitalo, E. (2012). Progressive sheet-to-tubule transformation is a general mechanism for endoplasmic reticulum partitioning in dividing mammalian cells. Mol. Biol. Cell 23, 2424-2432. doi: 10.1091/mbc.E10-12-0950

Rikihisa, Y. (2011). Mechanisms of obligatory intracellular infection with Anaplasma phagocytophilum. Clin. Microbiol. Rev. 24, 469-489. doi: 10.1128/CMR.00064-10

Roy, C. R., Salcedo, S. P., and Gorvel, J. P. (2006). Pathogen-endoplasmicreticulum interactions: in through the out door. Nat. Rev. Immunol. 6, 136-147. doi: $10.1038 /$ nri1775

Seidman, D., Hebert, K. S., Truchan, H. K., Miller, D. P., Tegels, B. K., Marconi, R. T., et al. (2015). Essential domains of Anaplasma phagocytophilum invasins utilized to infect mammalian host cells. PLoS Pathog. 11:e1004669. doi: 10.1371/journal.ppat.1004669

Sherwood, R. K., and Roy, C. R. (2013). A Rab-centric perspective of bacterial pathogen-occupied vacuoles. Cell Host Microbe 14, 256-268. doi: 10.1016/j.chom.2013.08.010
Shibutani, S. T., and Yoshimori, T. (2014). A current perspective of autophagosome biogenesis. Cell Res. 24, 58-68. doi: 10.1038/cr.2013.159

Stenmark, H. (2009). Rab GTPases as coordinators of vesicle traffic. Nat. Rev. Mol. Cell Biol. 10, 513-525. doi: 10.1038/nrm2728

Suarez, C. E., and Noh, S. (2011). Emerging perspectives in the research of bovine babesiosis and anaplasmosis. Vet. Parasitol. 180, 109-125. doi: 10.1016/j.vetpar.2011.05.032

Sukumaran, B., Mastronunzio, J. E., Narasimhan, S., Fankhauser, S., Uchil, P. D., Levy, R., et al. (2011). Anaplasma phagocytophilum AptA modulates Erk1/2 signalling. Cell. Microbiol. 13, 47-61. doi: 10.1111/j.1462-5822.2010. 01516.x

Swanson, M. S., and Isberg, R. R. (1995). Association of Legionella pneumophila with the macrophage endoplasmic reticulum. Infect. Immun. 63, 3609-3620.

Terasaki, M., Shemesh, T., Kasthuri, N., Klemm, R. W., Schalek, R., Hayworth, K. J., et al. (2013). Stacked endoplasmic reticulum sheets are connected by helicoidal membrane motifs. Cell 154, 285-296. doi: 10.1016/j.cell.2013.06.031

Tesh, M. J., Morse, S. A., and Miller, R. D. (1983). Intermediary metabolism in Legionella pneumophila: utilization of amino acids and other compounds as energy sources. J. Bacteriol. 154, 1104-1109.

Troese, M. J., and Carlyon, J. A. (2009). Anaplasma phagocytophilum densecored organisms mediate cellular adherence through recognition of human Pselectin glycoprotein ligand 1. Infect. Immun. 77, 4018-4027. doi: 10.1128/IAI. 00527-09

Troese, M. J., Kahlon, A., Ragland, S. A., Ottens, A. K., Ojogun, N., Nelson, K. T., et al. (2011). Proteomic analysis of Anaplasma phagocytophilum during infection of human myeloid cells identifies a protein that is pronouncedly upregulated on the infectious dense-cored cell. Infect. Immun. 79, 4696-4707. doi: 10.1128/IAI.05658-11

Truchan, H. K., Seidman, D., and Carlyon, J. A. (2013). Breaking in and grabbing a meal: Anaplasma phagocytophilum cellular invasion, nutrient acquisition, and promising tools for their study. Microbes Infect. 15, 1017-1025. doi: 10.1016/j.micinf.2013.10.010

Truchan, H. K., Viebrock, L., Cockburn, C. L., Ojogun, N., Griffin, B. P., Wijesinghe, D. S., et al. (2016). Anaplasma phagocytophilum Rab10-dependent parasitism of the trans-Golgi network is critical for completion of the infection cycle. Cell. Microbiol. 18, 260-281. doi: 10.1111/cmi.12500

VieBrock, L., Evans, S. M., Beyer, A. R., Larson, C. L., Beare, P. A., Ge, H., et al. (2014). Orientia tsutsugamushi ankyrin repeat-containing protein family members are Type 1 secretion system substrates that traffic to the host cell endoplasmic reticulum. Front. Cell. Infect. Microbiol. 4:186. doi: 10.3389/fcimb.2014.00186

Visser, E. S., McGuire, T. C., Palmer, G. H., Davis, W. C., Shkap, V., Pipano, E., et al. (1992). The Anaplasma marginale msp5 gene encodes a 19-kilodalton protein conserved in all recognized Anaplasma species. Infect. Immun. 60, 5139-5144.

Wamsley, H. L., Alleman, A. R., Johnson, C. M., Barbet, A. F., and Abbott, J. R. (2011). Investigation of endothelial cells as an in vivo nidus of Anaplasma marginale infection in cattle. Vet. Microbiol. 153, 264-273. doi: 10.1016/j.vetmic.2011.05.035

Woldehiwet, Z., Horrocks, B. K., Scaife, H., Ross, G., Munderloh, U. G., Bown, K., et al. (2002). Cultivation of an ovine strain of Ehrlichia phagocytophila in tick cell cultures. J. Comp. Pathol. 127, 142-149. doi: 10.1053/jcpa.2002.0574

Zivkovic, Z., Blouin, E. F., Manzano-Roman, R., Almazan, C., Naranjo, V., Massung, R. F., et al. (2009). Anaplasma phagocytophilum and Anaplasma marginale elicit different gene expression responses in cultured tick cells. Comp. Funct. Genomics 2009:705034. doi: 10.1155/2009/705034

Conflict of Interest Statement: The authors declare that the research was conducted in the absence of any commercial or financial relationships that could be construed as a potential conflict of interest.

Copyright (c) 2016 Truchan, Cockburn, Hebert, Magunda, Noh and Carlyon. This is an open-access article distributed under the terms of the Creative Commons Attribution License (CC BY). The use, distribution or reproduction in other forums is permitted, provided the original author(s) or licensor are credited and that the original publication in this journal is cited, in accordance with accepted academic practice. No use, distribution or reproduction is permitted which does not comply with these terms. 